\title{
System Identification of Active Magnetic Bearing for Commissioning
}

Shahbaz Abdul Khader 



\section{O \\ UPPSALA \\ UNIVERSITET}

Teknisk- naturvetenskaplig fakultet UTH-enheten

Besöksadress:

Ångströmlaboratoriet

Lägerhyddsvägen 1

Hus 4, Plan 0

Postadress:

Box 536

75121 Uppsala

Telefon:

$018-4713003$

Telefax:

$018-4713000$

Hemsida:

http://www.teknat.uu.se/student

\section{Abstract \\ System Identification of Active Magnetic Bearing for Commissioning}

Shahbaz Abdul Khader

Active magnetic bearing (AMB) is an ideal bearing solution for high performance and energy efficient applications. Proper operation of AMB can be achieved only with advanced feedback control techniques. An identified system model is required for synthesizing high performance model based controllers. System identification is the preferred method for obtaining an accurate model. Therefore, it becomes a prerequisite for the commissioning of AMB. System identification for commissioning poses some challenges and special requirements. In this thesis, system identification of $A M B$ is approached within the context of commissioning. A procedure for identification is developed and applied to experimental data from a prototype AMB system. The identification procedure is based on the so called prediction error method, and it has been performed in the frequency domain. A linear state-space model, along with the required parameters, is successfully identified.
Handledare: Bin Liu

Ämnesgranskare: Kristiaan Pelckmans

Examinator: Philipp Rümmer

IT 15002

Tryckt av: Reprocentralen ITC 



\section{Acknowledgements}

I would like to express my gratitude to my reviewer Kristiaan Pelckmans for the useful remarks, feedback and engagement through the learning process of this master thesis. I thank you for proofreading the manuscript.

This thesis project was carried out in ABB Corporate research, Västerås, Sweden. I thank all members, including the group manager Jonas Larsson, of the Mechatronics group for providing me the opportunity and the right environment for completing this thesis. Furthermore I would like to thank my supervisor Bin Liu for introducing me to AMB systems and his constant support throughout the project.

A special thanks goes to Johan Sjöberg, who showed a great deal of enthusiasm and willingness to help me with this project. I appreciate your effort and it has proven to be very valuable. 



\section{Table of Contents}

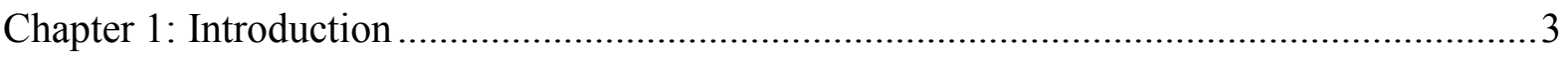

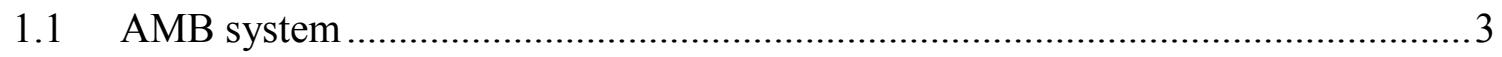

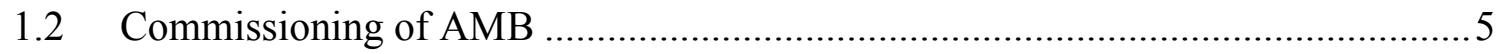

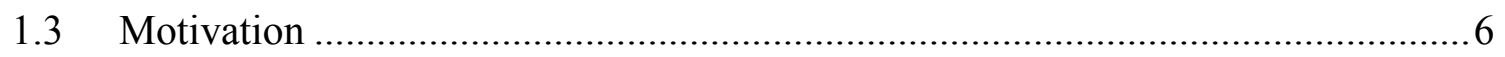

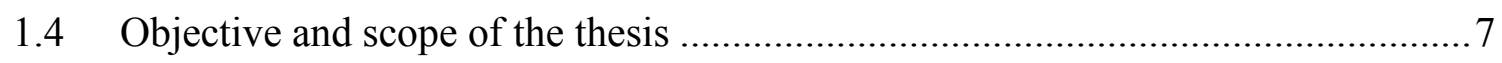

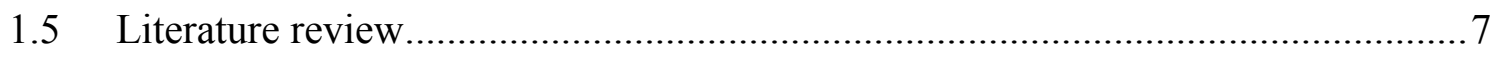

Chapter 2: Analytical Modeling of AMB systems .............................................................

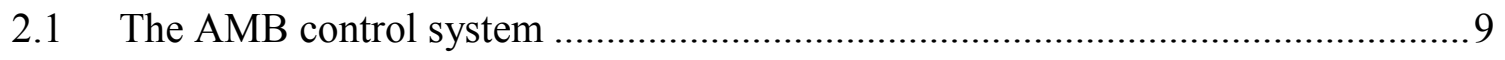

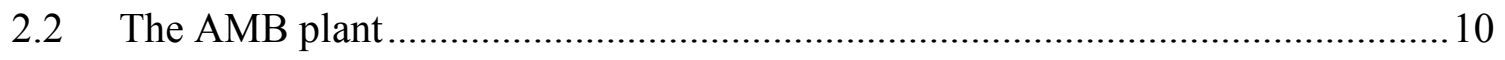

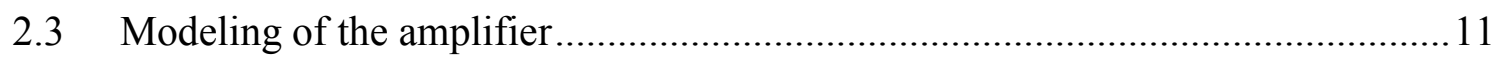

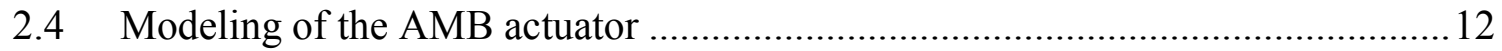

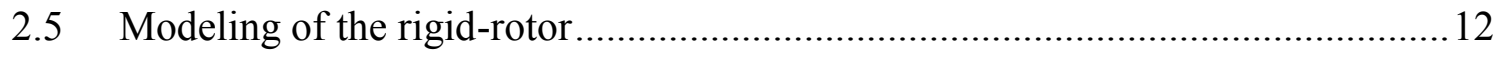

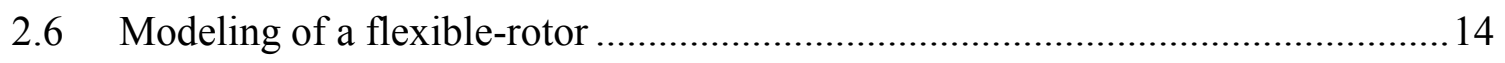

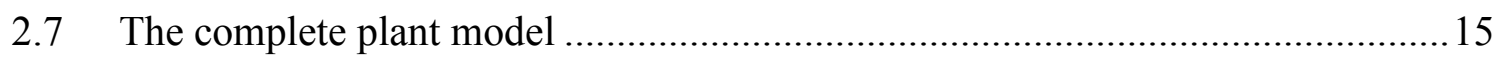

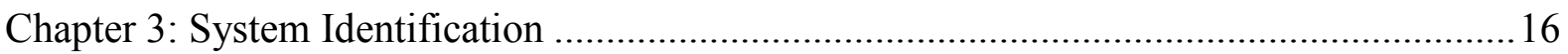

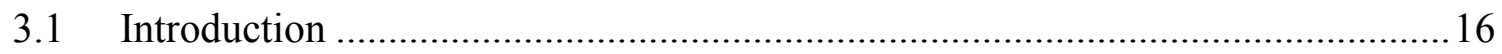

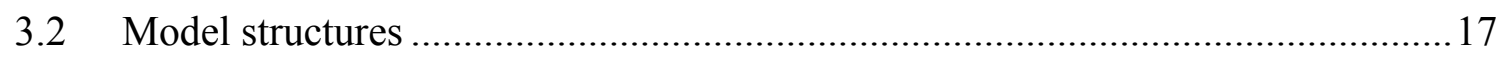

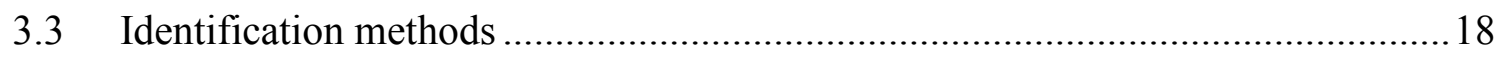

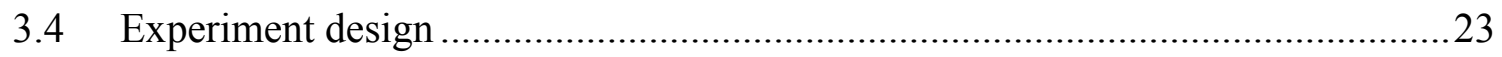

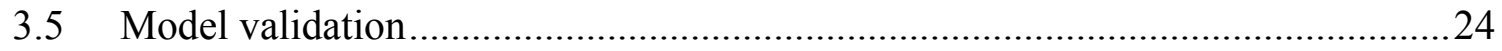

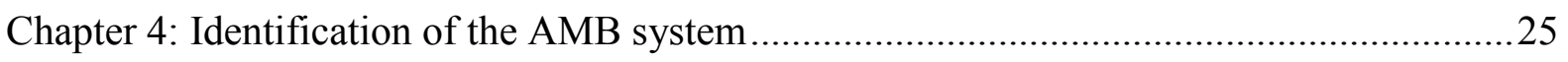

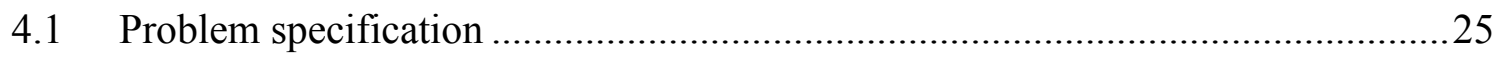




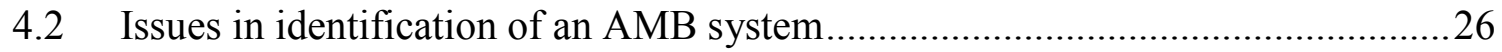

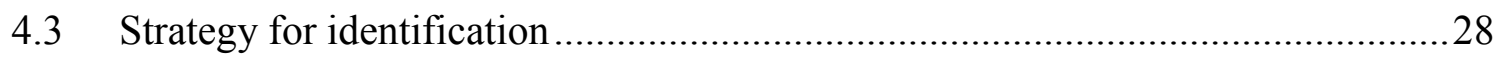

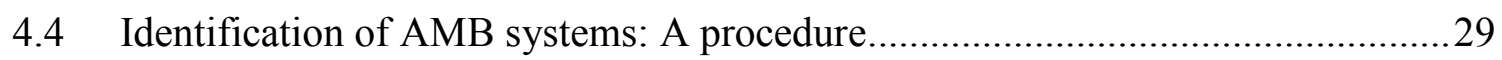

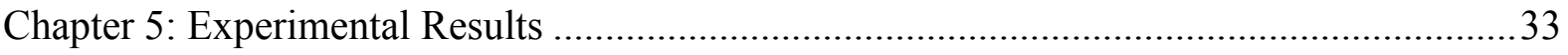

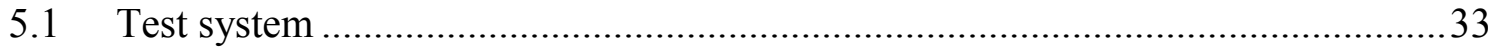

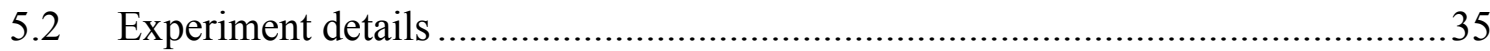

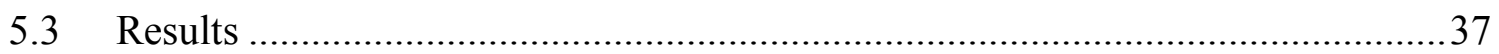

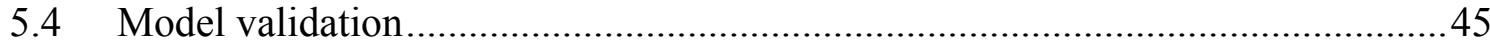

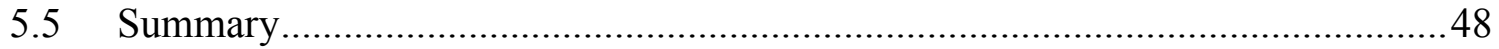

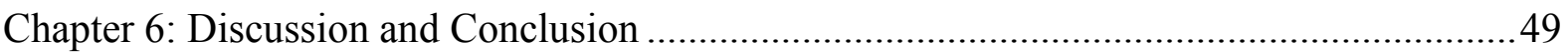

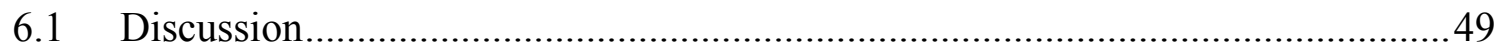

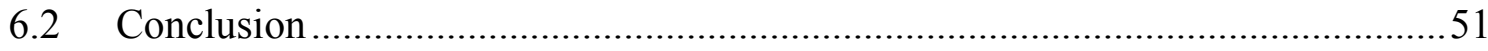

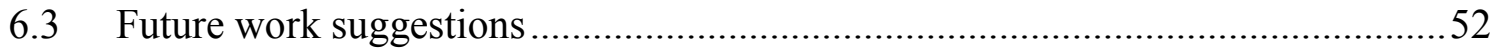

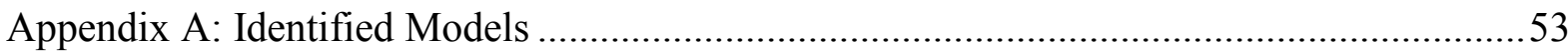

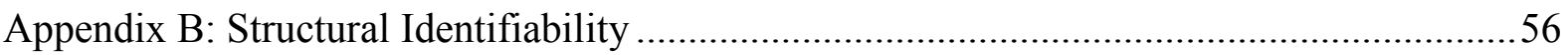

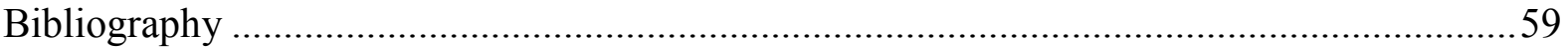




\section{Chapter 1}

\section{Introduction}

This chapter presents the background and motivation for the thesis. An overview of active magnetic bearing (AMB) system, their principle of operation and their typical applications are mentioned. System identification of AMB, its importance to system commissioning, and some earlier works, are discussed. Finally, the goal and scope of the thesis are stated.

\subsection{AMB system}

\subsubsection{AMB overview}

Magnetic bearings operate based on the phenomenon of magnetic levitation. Levitation eliminates mechanical contact between the bearing and the rotating shaft, and thereby achieves zeros friction. Magnetic bearings are available in two types, namely, passive magnetic bearing (PMB) and active magnetic bearing (AMB). Unlike AMBs, PMBs use permanent magnets and therefore do not require any controlling power inputs. But these systems are difficult to construct and offers no possibility to actively control their performance [1]. On the other hand, AMBs offer the possibility to actively control the rotor levitation by means of a pair of electromagnets. The possibility of active control allows a more relaxed construction specification for AMBs. For this reason, AMBs are widely preferred over PMBs. A comprehensive account of theory, operation and construction of AMB systems can be found in [1].

\subsubsection{Operating principle}

The basic operating principle of AMB systems can be explained with the help of Fig. 1.1. The current in the electromagnet produces a magnetic force which counteracts the force of gravity and enables the ferromagnetic ball to levitate. If the ball moves upwards from the equilibrium position and the current remains unchanged, the magnetic force increases and causes it to move up until it hits the top. Conversely, if the ball moves down, the magnetic force weakens and causes it to fall down. To avoid this unstable behavior an active closed loop control is necessary to maintain levitation. The controller regulates the position of the ball based on the measured position.

In practice, two counteracting electromagnets operating in differential driving mode are employed to control the rotor. Additionally, another pair of differential electromagnets is present to control the motion in horizontal direction. Such an arrangement constitutes a single AMB. In a typical AMB application the rotating shaft is supported by two AMBs at opposite ends of the rotor. Figure 1.2 shows a typical AMB system. The electromagnets are actuators of 
the system, and there are four of them in total. Each of the actuators has a corresponding position sensor which feeds back the positions of the rotor, in their respective planes (vertical or horizontal), back to the position controller. A closed loop control system is implemented in a microprocessor-based controller to regulate the positions of the rotor ends and thereby maintain levitation. The motor delivers mechanical power to the load through the rotor shaft.

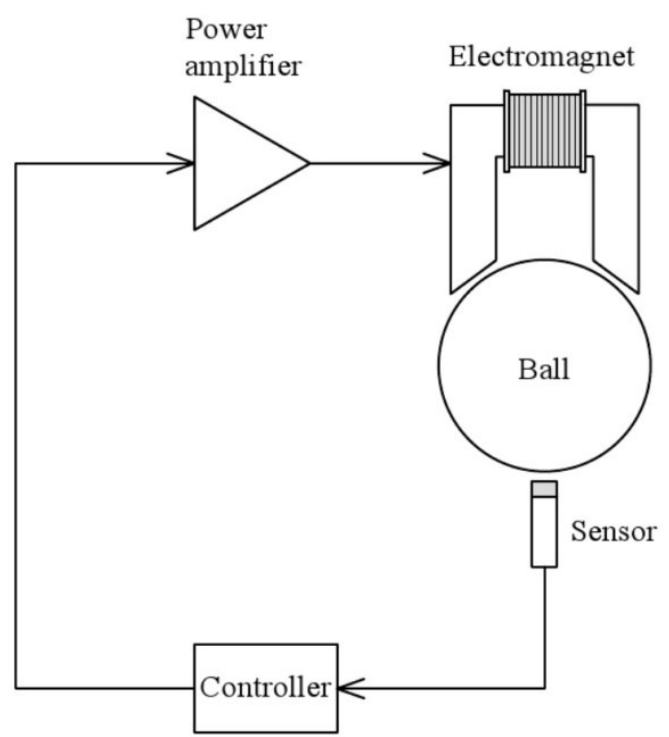

Figure 1.1 One degree-of-freedom AMB [6]

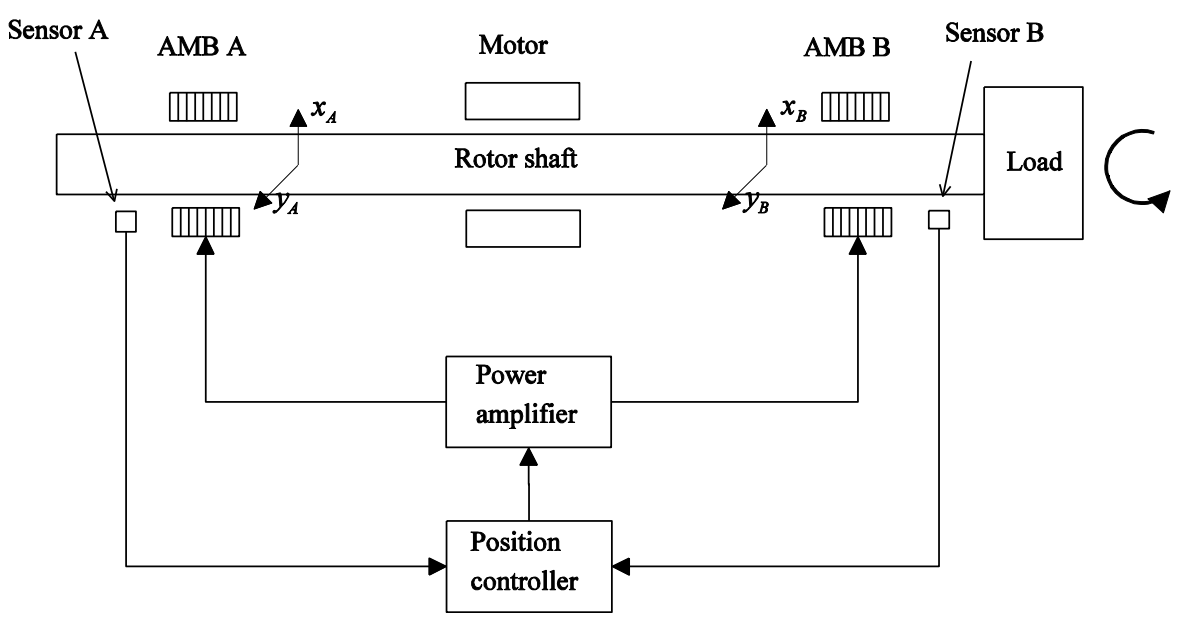

Figure 1.2 A typical AMB system. $x$ and $y$ represents the two orthogonal planes of motion.

\subsubsection{Applications of AMB}

The advantages of AMB are numerous: high speed, maintenance free (no wear and tear), energy efficiency (no friction), clean environment friendly (no lubricants), vibration-free (no mechanical contact) and active control of damping or stiffness. Not surprisingly there has been increasing application of AMB technology, especially for high speed applications such as gas turbines and centrifuges. Other common applications include compressors, pumps and tooling machines. 


\subsubsection{Control of AMB}

From a control perspective, the plant consists of the rotor, the load, the AMB actuator, and the power amplifier. AMBs are unstable, nonlinear and multiple-input multiple-output $(4 \times 4$ MIMO) systems. As mentioned earlier, closed loop control is a necessity for their operation. This has implications for system identification because the identification procedure can only be performed in the presence of a stabilizing closed loop. The goal of the controller is to sustain levitation and maintain the rotor position at the center of the stator (for each AMB). Controller synthesis for such a system is difficult and this constitutes the primary challenge in the operation of AMB. Several methods ranging from classical PID [2] to modern methods of LQG [3], $H \infty$ [4] and $\mu$-synthesis [5] have been applied to AMB. Other significant works include [6] and [7].

Modern methods are model based methods which require a model of the control plant. The higher the performance specifications, the more accurate the plant model must be.

\subsection{Commissioning of AMB}

\subsubsection{Complexities of AMB system}

A general characteristic of an AMB system is that it is a complex mechatronic system. Commissioning of such a system requires a fusion of multiple disciplines such as mechanical, control, electronics and software engineering. A recent work which dealt with commissioning is [8]. Commissioning of AMB systems involves some complex activities such as sensor/actuator tuning, controller synthesis, identification experiments, unbalance tuning and fault detection [8]. It requires highly skilled labor. All of these affect significantly the time and cost of commissioning.

\subsubsection{Robustness of control}

Achieving robust control of AMB systems is the key requirement of commissioning. Controller performance and robustness usually contradict each other, and the only way to improve both of them simultaneously is by synthesizing advanced model based controller based on an accurate plant model.

\subsubsection{Automated commissioning}

Automating the different steps of commissioning is an attractive and promising vision for the future of AMB. Automation can mitigate the requirement of highly skilled labor and significantly cut down on the commissioning time. This could open up the market for AMB in several application domains. Lösch presented a comprehensive method for automating controller synthesis in [6]. Smirnov looked at automating all aspects of commissioning in [8].

\subsubsection{System diagnostics}


Capability for system diagnosis during and after commissioning is an important requirement. Step by step verification during start up and regular health checkup of the system can save time and cost. It could also prevent potential accidents. Two related works are [9] and [10].

\subsection{Motivation}

\subsubsection{Analytical vs Experimental model}

Most high performance control systems utilize a plant model for controller synthesis. One way to obtain the required model is through analytical modeling, where the system is modelled mathematically using the laws of physics. Such a model would suffer from shortcomings such as unverified system parameters, linearization errors and various other approximations. An alternative to obtain the plant model is by system identification. In system identification the model is identified experimentally. A good identification procedure would provide a more accurate model compared to the analytical model. Therefore, system identification becomes a necessary and crucial part of AMB operation.

\subsubsection{Identification for commissioning}

Several methods have been researched and experimented for identification of AMB rotor systems over the years. Most of these works were for purely academic research, using experimental systems in the laboratory. Identification for commissioning could have some special considerations, of which some are discussed below.

Some identification methods require analytical models. An analytical model of a flexible rotor can be developed with the help of Finite Element Modeling (FEM). However, from a commissioning point of view, FEM is a time consuming and expensive proposition which involves highly skilled labor. Identification for commissioning should be independent of FEM.

The identification process itself must be robust against the relatively high noise in the commissioning site. This could be achieved by designing excitation signals to be of high signal to noise ratio (SNR).

Finally, the identification procedure should be able to identify certain system parameters for diagnostics or verification purposes. This should also mean that the procedure must be more or less automated, and should be independent of any external equipment or devices. The latter is usually the case with most AMB systems, since the same controller, sensors and actuators which are used for the normal operation of AMB can also be used for identification.

To summarize, identification of AMB should have the following requirements:

1. Identify an accurate system model.

2. Identify a set of special system parameters.

3. The process should be robust against noise. 
4. The procedure should be independent of FEM.

5. The procedure should be automated as well as possible.

If these requirements are met, it would significantly reduce the time and cost for the commissioning of AMB systems.

\subsection{Objective and scope of the thesis}

\subsubsection{Background}

This thesis is a part of AMB research \& development activity by Asea Brown Boveri (ABB) in their Corporate Research facility (CRC) in Västerås, Sweden. The AMB development team in $\mathrm{ABB}$ has already developed an experimental AMB system. An analytical model of the system has been completed and a PID based controller has been synthesized using a novel optimization procedure [11]. A successful levitation has been achieved. The broad objective of the project is to develop affordable and efficient commissioning. Hence, system identification is the next logical step for this project.

\subsubsection{Problem statement}

- Goal: To develop and verify an identification procedure that meets the requirements for commissioning.

- Scope: Limited to nonrotating rotor only. Rotating rotor could introduce gyroscopic effects, but this thesis does not aim to consider it.

\subsection{Literature review}

System identification of AMB system stretches back to early nineties. System identification, in general, includes both nonparametric (frequency response) and parametric (state-space or transfer function models) identification. It could be done in either time domain or frequency domain. However, most of the work done towards identification of AMB systems is in frequency domain. For time-domain identification, nonparametric models are not really necessary but are usually a useful starting point. The most important works for AMB identification are reviewed in this section.

Identification in frequency domain is usually done in two steps: Firstly, a nonparametric model (or frequency response function - FRF) is obtained by performing an appropriate experiment. The most common nonparametric methods are reviewed and compared in [12] and [13]. Secondly, a frequency domain fit between a parameterized model structure (obtained from the analytical model) and the nonparametric model is performed. PEM, for most model structures, leads to a nonlinear optimization problem. The result of the fit will be the unknown parameters in the model structure. A detailed analysis of existing methods for this approach is given in [13]. 
One of the earliest works in frequency domain was done by Gähler et al. [14], in which the authors invented a unique method for identifying poles of the transfer function. The poles of the system were first estimated from the determinant of the measured frequency response functions, and then the remaining parameters are estimated by a sequence of linear least squares problems. This method was later used successfully by Lösch [6] and with limited success by Hynynen [12]. All three of them used a MIMO adaptation of Sanathanan-Koerner's (SK) algorithm [15], which is an iterative weighted linear square estimation method for transfer function models, for solving the resulting nonlinear optimization problem. Hynynen also tried a more general method based on Matrix Fraction Description (MFD) representation. A similar approach, but based on instrumental variable (IV) method, was presented by Blom et al. [16]. The authors claim that, unlike SK, convergence implies a stationary point of the cost function. Another way to solve the nonlinear optimization problem is to directly solve it in MATLAB with one of the standard algorithms. This was done by Ahn et al. [17] and Balini et al. [18]. This approach can only work if a good initial estimate of the parameters is already known (to avoid local minima). This is usually achieved by a preliminary identification step such as time domain subspace method (Balini et al.) or SK algorithm (Ahn et al). Ahn et al. also developed 'control relevant identification' which involves iterative design of controller and updating the model parameters. A frequency domain subspace method was developed by Mokhtar et al. in [19].

Identification of AMB systems in the time domain is almost exclusively based on subspace methods. Balini et al. in [18] used a recent advancement known as predictor based subspace identification (PBSID ${ }_{\mathrm{OPT}}$ ) [20], which is specially geared for identification in closed loop. However, subspace identification is not guaranteed to be optimal and therefore they used the result from subspace method as an initial estimate for the subsequent frequency-domain identification. Such a cascading of subspace method with PEM is a recommended strategy [21]. Another work which uses a subspace method is presented in [22] by Cho et al., in which the plant is considered as a set of uncoupled SISO systems, but the authors fail to highlight the challenges of subspace identification in closed loop.

Most of these works are not focused on commissioning. Only Lösch provided an automated procedure for identification (and controller synthesis), but did not identify AMB stiffness constants. Smirnov, in [8], presented a procedure for commissioning, but did not provide details of the identification method. 


\section{Chapter 2}

\section{Analytical Modeling of AMB systems}

In this chapter, an analytical model of the AMB system is presented. The approach which is taken is to model each individual component of the system, namely, the amplifier, the electromagnetic actuator and the rotor. These models can then be combined to form a complete model of the plant. During this process the system is linearized and, therefore, a linear statespace model is the end result. The model obtained in this chapter will be used as the model structure for system identification.

\subsection{The AMB control system}

The operating principle of AMB has been presented in Sec. 1.1.2. Figure 2.1 shows a more detailed schematic of a single AMB. As shown in the figure, the controller senses the displacement in both $x$ and $y$ planes. This constitutes the feedback to the control system. The controller generates a control signal, $u$, based on the control law. This signal is added/subtracted to a bias current, $u_{b}$, and supplied to the top/bottom amplifier of the corresponding plane $(x$ or $y$ ). Such a configuration of a pair of electromagnets is called a differential driving mode, and together constitutes a single actuator. The constant bias current $u_{b}$ is required to magnetize the electromagnet. Each of the amplifier unit is a closed-loop current controller. Their function is to develop current in the electromagnetic coil to match the control signal. The two orthogonal planes $x$ and $y$ are both inclined by $45^{\circ}$ to the horizontal direction in order to distribute the effect of gravity equally between them.

In practice, two AMBs are required to levitate a rotor (Fig.1.2). The $x / y$ directions of the two AMBs are aligned perfectly. In each plane there are two actuators, one at side A and the other at side B. The AMBs will be referred to as AMB A and AMB B, hereafter. Each of the electromagnetic actuator has a corresponding position sensor which senses the displacement of the rotor. These will be referred to as sensor A and sensor B. In addition to the four actuator-sensor pairs, there is another actuator-sensor pair for the control of the rotor in axial direction. This is treated separately and will not be considered in this thesis. The system consists of four inputs and four outputs, rendering it a $4 \times 4$ MIMO system. If only one of the planes were to be considered, then the system will be a $2 \times 2$ MIMO system. Fig 2.2 illustrates the overall control system of AMB. 


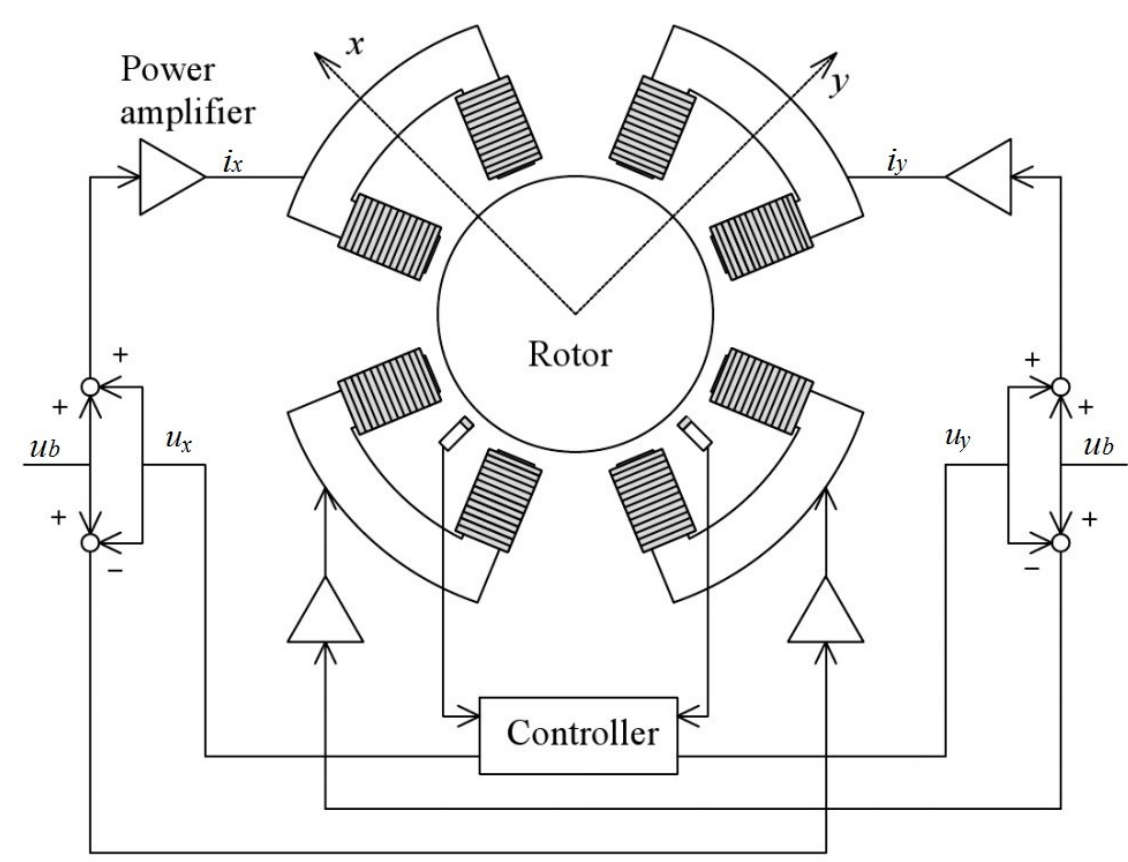

Figure 2.1 Operational principle of an AMB. The figure illustrates a cross-sectional view of the AMB-rotor assembly. $x$ and $y$ are the two orthogonal planes of motion. $\boldsymbol{u}_{\boldsymbol{x}} / \boldsymbol{u}_{\boldsymbol{y}}$ is the current control signal, $\boldsymbol{u}_{\boldsymbol{b}}$ is the bias current and $\boldsymbol{i}_{\boldsymbol{x}} / \boldsymbol{i}_{\boldsymbol{y}}$ is the coil current developed by the amplifier in the coil. $\boldsymbol{u}$ is applied to the top and bottom electromagnets in differential mode (Adapted from [6]).

\subsection{The AMB plant}

From a control perspective, the plant consists of three components, viz. amplifier, AMB actuator, and rotor. Figure 2.2 illustrates the overall control system. This implies that the signals r, u, i and f in Fig. 2.2 are defined as follows.

$\mathrm{r}=\left[\begin{array}{llll}r_{x A} & r_{x B} & r_{y A} & r_{y B}\end{array}\right]^{T} \quad$ reference input (ampere, A)

$\mathrm{u}=\left[\begin{array}{llll}u_{x A} & u_{x B} & u_{y A} & u_{y B}\end{array}\right]^{T} \quad$ input to the plant, control current signal (ampere, $\mathrm{A}$ )

$\mathrm{y}=\left[\begin{array}{llll}y_{x A} & y_{x B} & y_{y_{A}} & y_{y_{B}}\end{array}\right]^{T} \quad$ displacement of the rotor ends, output of the system (meter, $m$ )

$\mathrm{i}=\left[\begin{array}{llll}i_{x A} & i_{x B} & i_{y A} & i_{y B}\end{array}\right]^{T} \quad$ current formed in the AMB coil (ampere, A)

$\mathrm{f}=\left[\begin{array}{llll}f_{x A} & f_{x B} & f_{y A} & f_{y B}\end{array}\right]^{T} \quad$ force generated by the AMB (Newton, $\mathrm{N}$ )

where the subscript $x / y$ refers to the plane of motion, and $A / B$ refers to the rotor end.

It is a known fact that the dynamics of motion in planes $x$ and $y$ are completely decoupled, if gyroscopic effects are ignored [1]. Since this is the case for the thesis, modeling and identification of the system in individual planes can be carried out independently. Therefore, the modeling and identification problem is reduced to a $2 \times 2$ MIMO system. Hereafter, only one plane will be discussed and for rest of the report the following definition of the control variables apply.

$\mathrm{r}=\left[\begin{array}{ll}r_{A} & r_{B}\end{array}\right]^{T}$ 
$\mathrm{u}=\left[\begin{array}{ll}u_{A} & u_{B}\end{array}\right]^{T}$

$\mathrm{y}=\left[\begin{array}{ll}y_{A} & y_{B}\end{array}\right]^{T}$

$\mathrm{i}=\left[\begin{array}{ll}i_{A} & i_{B}\end{array}\right]^{T}$

$\mathrm{f}=\left[\begin{array}{ll}f_{A} & f_{B}\end{array}\right]^{T}$

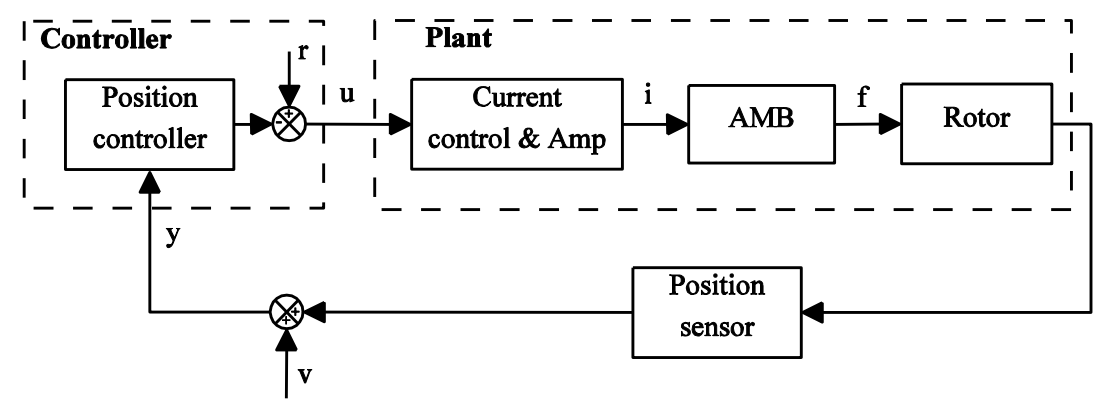

Figure 2.2 A block diagram of AMB control system. The plant consists of power amplifier, $\mathrm{AMB}$ actuator and rotor. Vectors $\mathbf{r}, \mathbf{u}, \mathbf{i}, \mathbf{f}$ and $\mathbf{y}$ represent four dimensional reference input, plant input (control current), coil current, magnetic force, and plant output (rotor displacement), respectively. The additive noise is represented by $\mathbf{v}$.

\subsection{Modeling of the amplifier}

The amplifier model accepts the voltage signal from the controller and outputs the current signal to the actuator coil. It does so by employing a closed loop control of its own. The current control is usually a pure proportional control. Under the assumption that the coil resistance is small and the inductance is constant, the following transfer function is obtained [1]

$$
G_{A}(s)=\frac{K_{p}}{s L+K_{p}}=\frac{\omega_{b w}}{s+\omega_{b w}}
$$

where $K_{p}$ is the proportional gain of the $\mathrm{P}$ control and $L$ is the coil inductance. The quantity $\omega_{b w}$ is the bandwidth of the first order system. More details about amplifier modeling can be found in [1] and [6].

The above mentioned transfer function is for a single amplifier. While considering a single plane, the $2 \times 2$ MIMO model for the amplifier can be expressed in state space from as follows.

$$
\begin{gathered}
\dot{\mathrm{x}}_{\mathrm{a}}=\mathrm{A}_{\mathrm{a}} \mathrm{x}_{\mathrm{a}}+B_{a} \mathrm{u} \\
\mathrm{i}=\mathrm{C}_{\mathrm{a}} \mathrm{x}_{\mathrm{a}}+\mathrm{D}_{\mathrm{a}} \mathrm{u} \\
\mathrm{x}_{\mathrm{a}}=\left[\begin{array}{l}
i_{A} \\
i_{B}
\end{array}\right], \mathrm{u}=\left[\begin{array}{l}
u_{A} \\
u_{B}
\end{array}\right], \mathrm{A}_{\mathrm{a}}=\left[\begin{array}{cc}
-\omega_{b w} & 0 \\
0 & -\omega_{b w}
\end{array}\right], \mathrm{B}_{\mathrm{a}}=\left[\begin{array}{cc}
\omega_{b w} & 0 \\
0 & \omega_{b w}
\end{array}\right], \mathrm{C}_{\mathrm{a}}=\left[\begin{array}{ll}
1 & 0 \\
0 & 1
\end{array}\right], \mathrm{D}_{\mathrm{a}}=[0]
\end{gathered}
$$

The amplifier model can be identified independently if the current signal ' $i$ ' is measured. 


\subsection{Modeling of the AMB actuator}

Referring to the block diagram in Fig. 2.2, the AMB actuator accepts the coil current signal and outputs magnetic force. Electromagnetism introduces nonlinearity into the system at this stage. However, it has been found that the system can be linearized around an operation point using the following model [1], [6]

$$
f=k_{i} i+k_{s} x
$$

where $x$ is the rotor displacement, and $k_{i}, k_{s}$ are two constants called current stiffness and position stiffness constants, respectively. $i$ and $f$ are coil current and force respectively.

The stiffness constants are defined as:

$$
\begin{array}{r}
k_{i}=\frac{4 k u_{b}}{s_{0}^{2}}, \quad k_{s} \\
=-\frac{4 k u_{b}^{2}}{s_{0}^{3}}
\end{array}
$$

where $u_{b}$ is a suitable bias current, $s_{0}$ is the corresponding position of the rotor, preferably at the center of the air gap and $k$ is a constant derived from mechanical and magnetic construction. The pair $s_{0}, u_{b}$ defines an appropriate operation point around where the model is assumed to be linear. Practical experience has shown that this linear model works very well [12]. This linear model also reveals the cause of the inherent instability of the system, namely the negative position stiffness, $k_{s}$. A small disturbance from the equilibrium position would cause an uncontrolled fall of the rotor to either the top or bottom magnets. Hence the AMB system is open-loop unstable and can be stabilized only with closed-loop control.

The actuator model for the system can be written as

$$
\mathrm{F}=\mathrm{K}_{\mathrm{i}} \mathrm{i}+\mathrm{K}_{\mathrm{s}} \mathrm{x}
$$

where $\mathrm{F}=\left[\begin{array}{cc}f_{A} & 0 \\ 0 & f_{B}\end{array}\right], \mathrm{K}_{\mathrm{S}}=\left[\begin{array}{cc}k_{S A} & 0 \\ 0 & k_{s B}\end{array}\right]$ and $\mathrm{K}_{\mathrm{i}}=\left[\begin{array}{cc}k_{i A} & 0 \\ 0 & k_{i B}\end{array}\right]$ are the force, position stiffness and current stiffness matrices respectively. $\mathrm{x}=\left[\begin{array}{l}x_{A} \\ x_{B}\end{array}\right]$ is the rotor displacement.

\subsection{Modeling of the rigid-rotor}

In each of the planes, the rotor exhibits rotation about its center of gravity as well as a transversal movement. These are the two rigid modes of the rotor dynamics model. A subcritical AMB rotor system, one in which the range of operation speed does not cause 
excitation of flexible eigenfrequencies, can be modelled with a rigid-rotor model [1]. A very detailed discussion of rigid-rotor modeling can be found in [6]. Each of the two rigid modes is a second order mode with real valued pole pair, located symmetrically about the imaginary axis. The positive pole indicates instability.

The force balance equation of the actuator-rotor system can be written as

$$
\mathrm{M} \ddot{\mathrm{x}}=\mathrm{F}
$$

Combining (2.6) and (2.5),

$$
M \ddot{x}-K_{s} x=K_{i} i
$$

where,

$\mathrm{x}=\left[\begin{array}{l}x_{A} \\ x_{B}\end{array}\right]$ is the rotor displacements at the bearing locations, $\mathrm{M}=\left[\begin{array}{ll}m_{1} & m_{3} \\ m_{3} & m_{2}\end{array}\right]$ is the mass matrix.

The parameters $m_{1}, m_{2}$ and $m_{3}$ are functions of physical properties of the rotor, such as its mass $(m)$, transversal moment of inertia $\left(I_{r}\right)$, and distances $d_{A}$ and $d_{B}$ as defined in Fig. 2.3.

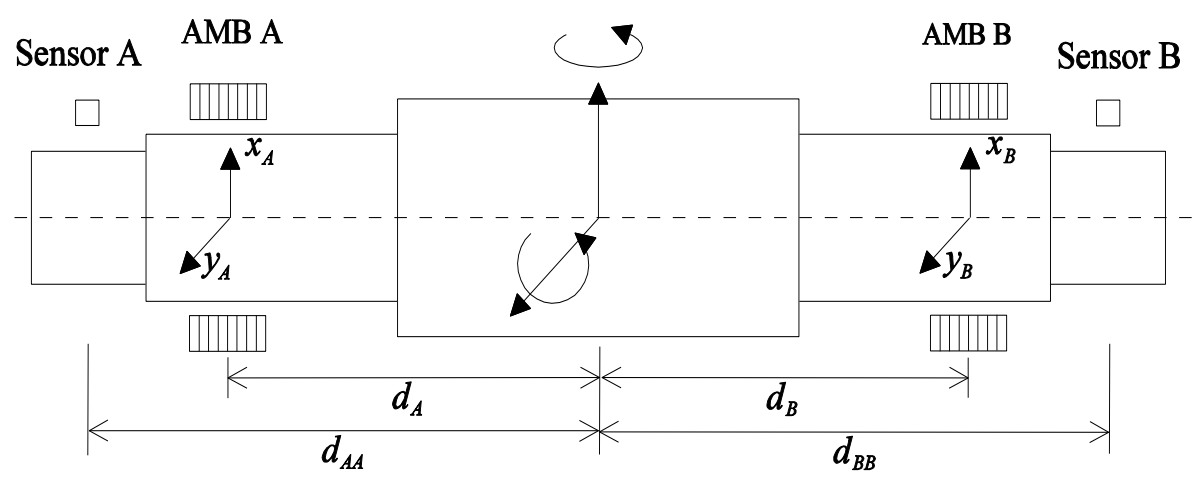

Figure 2.3. A drawing showing the physical dimensions of a rotor supported by two AMBs. The forces and displacements pertaining to the rotor are resolved along the two orthogonal planes $\boldsymbol{x}$ and $\boldsymbol{y}$. Bearings A and B are separated from the center of gravity of the rotor by $\boldsymbol{d}_{\boldsymbol{A}}$ and $\boldsymbol{d}_{\boldsymbol{B}}$. The sensors are separated from the same by $\boldsymbol{d}_{\boldsymbol{A} A}$ and $\boldsymbol{d}_{\boldsymbol{B} B}$.

The actuator-rotor model can be expressed in state-space form as

$$
\begin{gathered}
\dot{x}_{\mathrm{r}}=\mathrm{A}_{\mathrm{r}} \mathrm{x}_{\mathrm{r}}+\mathrm{B}_{\mathrm{r}} \mathrm{i} \\
\mathrm{y}=\mathrm{C}_{\mathrm{r}} \mathrm{x}_{\mathrm{r}}+\mathrm{D}_{\mathrm{r}} \mathrm{i}
\end{gathered}
$$




$$
A_{r}=\left[\begin{array}{cc}
0 & I \\
M^{-1} K_{s} & 0
\end{array}\right], B_{r}=\left[\begin{array}{c}
0 \\
M^{-1} K_{i}
\end{array}\right], C_{r}=\left[\begin{array}{ll}
T_{s} & 0
\end{array}\right], D_{r}=[0]
$$

where $\mathrm{x}_{\mathrm{r}}=\left[\begin{array}{l}\mathrm{x} \\ \dot{\mathrm{x}}\end{array}\right]$ and $\mathrm{T}_{\mathrm{S}}=\left[\begin{array}{cc}d_{A A} / d_{A} & 0 \\ 0 & d_{B B} / d_{B}\end{array}\right]$ is the output matrix. The full derivation and modeling can found in [6].

\subsection{Modeling of a flexible-rotor}

If the system operates above the critical frequency (first bending mode of the rotor), flexible modes of the rotor up to the operation frequency have to be modeled. Up to three flexible modes are not uncommon; however this depends on several factors such as mechanical characteristics, load on the rotor, observability and/or controllability of the modes etc. The system designer should decide the number of flexible modes before the modeling process. A flexible-rotor model is usually developed through finite element modeling (FEM). The number of flexible modes is controlled by the choice of number of nodes in FEM.

The force equation of a flexible rotor is as follows [6]

$$
M \ddot{q}+D \dot{q}+K q=f
$$

where matrices $\mathrm{M}, \mathrm{D}$ and $\mathrm{K}$ represent mass, internal damping and internal stiffness coefficients of the rotor, respectively, in modal coordinates. These are obtained from the finite element model (FEM). The variable q represents the nodal displacement vector.

Combining (2.9) and (2.5),

$$
M \ddot{q}+D \dot{q}+\left(K+K_{s}\right) q=K_{i} \dot{1}
$$

$\mathrm{K}_{\mathrm{s}}$ and $\mathrm{K}_{\mathrm{i}}$, in this case, represent the AMB stiffness constants in modal coordinates. The size of the nodal displacement vector $\mathrm{q}$, and hence all other matrices, depends on the number of nodes in FEM, which is usually limited to a number that is just enough to include the required number of eigenmodes. An overview of FEM for AMB systems can be found in [6].

The state-space model of the actuator-rotor (flexible) is as follows [6]

$$
\begin{gathered}
\dot{\mathrm{q}}_{\mathrm{f}}=\mathrm{A}_{\mathrm{f}} \mathrm{q}_{\mathrm{f}}+\mathrm{B}_{\mathrm{f}} \mathrm{i} \\
\mathrm{y}=\mathrm{C}_{\mathrm{f}} \mathrm{q}_{\mathrm{f}}+\mathrm{D}_{\mathrm{f}} \mathrm{i} \\
\mathrm{A}_{\mathrm{f}}=\left[\begin{array}{cc}
0 & \mathrm{I} \\
\mathrm{M}^{-1}\left(\mathrm{~K}-\mathrm{K}_{\mathrm{s}}\right) & -\mathrm{M}^{-1} \mathrm{D}
\end{array}\right], \mathrm{B}_{\mathrm{f}}=\left[\begin{array}{c}
0 \\
\mathrm{M}^{-1} \mathrm{~K}_{\mathrm{i}}
\end{array}\right], \mathrm{C}_{\mathrm{f}}=\left[\begin{array}{ll}
T_{m} & 0
\end{array}\right], \mathrm{D}_{\mathrm{f}}=[0]
\end{gathered}
$$


where $\mathrm{q}_{\mathrm{f}}=\left[\begin{array}{l}\mathrm{q} \\ \dot{\mathrm{q}}\end{array}\right]$ and $T_{m}$ is an appropriate output matrix.

\subsection{The complete plant model}

The complete plant model is obtained by combining the rigid or flexible rotor model with the amplifier model. The choice between a rigid or a flexible model depends on the application.

\subsubsection{The rigid-rotor model}

The complete plant model with rigid rotor is obtained by combining (2.8) and (2.2).

$$
\begin{gathered}
\dot{x}=A_{p} x+B_{p} u \\
y=C_{p} x+D_{p} u \\
A_{p}=\left[\begin{array}{cc}
A_{a} & 0 \\
B_{r} C_{a} & A_{r}
\end{array}\right], B_{p}=\left[\begin{array}{c}
B_{a} \\
0
\end{array}\right], C_{p}=\left[\begin{array}{ll}
0 & C_{r}
\end{array}\right], D_{p}=[0] \\
x=\left[\begin{array}{c}
x_{a} \\
x_{r}
\end{array}\right]
\end{gathered}
$$

\subsubsection{The flexible-rotor model}

The complete plant model with flexible rotor is obtained by combining (2.11) and (2.2).

$$
\begin{gathered}
\dot{x}=A_{p} x+B_{p} u \\
y=C_{p} x+D_{p} u \\
A_{p}=\left[\begin{array}{cc}
A_{a} & 0 \\
B_{f} C_{a} & A_{f}
\end{array}\right], B_{p}=\left[\begin{array}{c}
B_{a} \\
0
\end{array}\right], C_{p}=\left[\begin{array}{ll}
0 & C_{f}
\end{array}\right], D_{p}=[0] \\
x=\left[\begin{array}{c}
x_{a} \\
q_{f}
\end{array}\right]
\end{gathered}
$$




\section{Chapter 3}

\section{System Identification}

This chapter reviews the basic theory of system identification. It begins with an intuitive explanation of system identification, proceeds with discussion of the steps involved, and explains the various methods of identification. This chapter also includes details of experiment design and concludes with model validation techniques.

\subsection{Introduction}

System identification is the problem of experimentally determining the mathematical model of a dynamic system using its measured input-output data. Consider the system in Fig. 3.1, where a linear time-invariant (LTI) plant $G$ is subjected to a control input $u$ and outputs the signal $y$. Let $v$ be the additive noise of the system. Further, $v$ can be represented as a stochastic process i.e. filtered white noise, where $H$ is the noise dynamics and $e$ is white noise. System identification, more specifically, is the problem of finding $G$ and $H$ given $u$ and $y$.

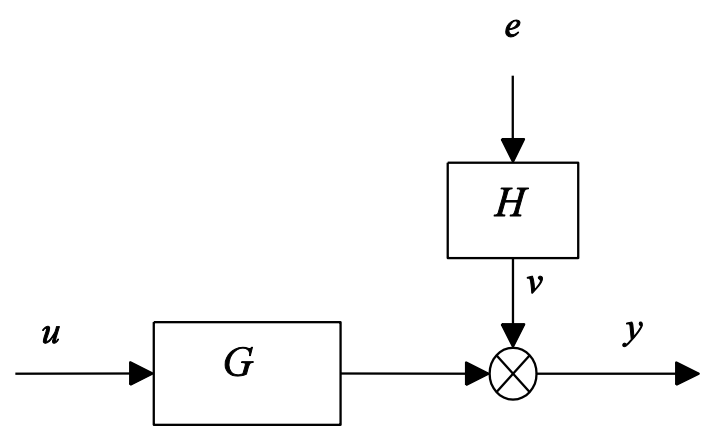

Figure 3.1. A system with additive noise

System identification requires three things, viz. an input-output data set $Z^{N}$ ( $u$ and $y$ ), a model structure $\mathcal{M}$ (for $G$ and $H$ ), and finally an identification method. The identification method tries to optimize the free parameters in $\mathcal{M}$ to achieve the best fit between the data set and the selected model structure. The steps involved in system identification are:

1. Experiment design.

2. Generate data set through experiments.

3. Choose a suitable model structure.

4. Obtain the best model in the model set using an appropriate identification method.

5. Validate the model. 
A thorough treatment of the subject can be found in [21] and [23].

\subsection{Model structures}

System identification involves a search for the best model among a set of models. The set of models is called the model structure and is denoted by $\mathcal{M}(\theta)$. The model set is parameterized with a parameter vector, denoted by $\theta$. A particular model within the set is denoted by $\mathcal{M}\left(\theta^{*}\right)$.

Mathematically, the system in Fig. 3.1 can be written as

$$
y(t)=G(p, \theta) u(t)+H(p, \theta) e(t) .
$$

where $p$ is the differential operator and $\theta$ is the parameter vector which parameterizes the transfer functions $G$ and $H$.

For such a formulation, the model structure can be written formally as

$$
\mathcal{M}(\theta): \quad \mathcal{D}_{\mathcal{M}} \ni \theta^{*} \rightarrow \mathcal{M}\left(\theta^{*}\right)=\left\{G\left(., \theta^{*}\right), H\left(., \theta^{*}\right)\right\}
$$

meaning, a mapping from $\theta^{*}$, a member of some parameter set $\mathcal{D}_{\mathfrak{M}}$, to a particular model $\mathcal{M}\left(\theta^{*}\right)$ within the model structure $\mathcal{M}(\theta)$ [13].

One of the first choices to make is to decide between linear and nonlinear model structures. Since the AMB model is linearized, nonlinear models will not be considered in this thesis. Model structures are defined by the order of the system and a specific parameterization. Transfer function model structures can be specified by choosing the number of poles and zeros. For state-space model structures, this is done by fixing the number of state variables and the form of the model, namely, modal, observer or controller canonical forms. Completely free parameterization of all matrix elements is also possible. In all of these cases the parameters may not be physically meaningful. Such models are called black-box models. If, on the other hand, the parameterization is such that the parameters are physically meaningful, it is called a grey-box model [21].

The selection of model structure is the most important and the most difficult part in system identification [21]. It is the user's responsibility to choose the model structure wisely. If the system can be modeled mathematically, using laws of physics, then such a model could give a good model structure.

\subsubsection{State-space model structures}

Equation (3.1) is in the form of a transfer function, but MIMO systems are best represented in state-space models (innovation form)

$$
\dot{x}(t)=A(\theta) x(t)+B(\theta) u(t)+K(\theta) e(t)
$$




$$
y(t)=C(\theta) x(t)+D(\theta) u(t)+e(t)
$$

where the state-space matrices are parameterized with an appropriate parameter vector $\theta$. Both representations are equivalent and the relationship between them is

$$
\begin{aligned}
& G(p, \theta)=C(\theta)(p I-A(\theta))^{-1} B(\theta)+D(\theta) \\
& H(p, \theta)=C(\theta)(p I-A(\theta))^{-1} K(\theta)+I
\end{aligned}
$$

where $K$ is the Kalman gain.

\subsubsection{Structural identifiablity}

While dealing with model structures, an important concept is structural identifiability. Let a parameterized model structure be represented by $\mathcal{M}(\theta)$, where $\theta$ is the parameter vector. The model structure $\mathcal{M}(\theta)$ is globally identifiable at $\theta^{*}$ if the equivalence $\mathcal{M}(\theta) \equiv \mathcal{M}\left(\theta^{*}\right)$ implies $\theta=\theta^{*}, \forall \theta \in \mathscr{D}_{\mathcal{M}}$. This concept is called structural identifiability. What it means, intuitively, is that the model cannot be reliably identified if the model is identical for more than one unique value of the parameter vector. If the structure is identifiable when $\theta$ is confined to a local neighborhood, then it is locally identifiable. More on identifiability can be found in [21] and [24].

\subsection{Identification methods}

The purpose of identification methods is to identify the parameter vector $\theta$ such that the error between the model and the data is minimized. But a parameterized model is not always the goal. Nonparametric models, which are impulse response functions or frequency response functions (FRF) at a large number of points, could also be identified from the time sampled input-output data. This divides identification methods into two classes, nonparametric and parametric methods. Nonparametric models are not parameterized functions, but rather numerical values at large number of time or frequency points. They are often a good starting point to get insight into the system order or quality of measurements. For frequency-domain parametric identification methods, nonparametric model (FRF) could be used as the data set, in which case it becomes a necessary step.

\subsubsection{Time-domain versus Frequency-domain methods}

One of the main decisions to make in system identification is to choose between time-domain and frequency-domain identification. The choice is easy if the system to be identified is nonlinear, since only linear systems can be easily represented in the frequency domain. Timedomain methods dominate the control and signal processing community, but frequency-domain methods continue to be used in measurement and instrumentation community [21]. A comprehensive literature on frequency-domain identification is Pintelon and Schoukens (2001) [23], while the most widely referred work for time-domain identification is Ljung (1999) [21]. 
A comparison between the two domains can be found in Pintelon and Schoukens (2001, pp. 368-373) and Ljung (1999, 227-233).

Time and frequency domain data are equivalent and the duality between them have emerged recently [25]. The primary issue with frequency domain methods, and the reason it got a bad name, is spectral leakage while transforming time-domain data into frequency domain (Discrete Fourier Transform or DFT) [23]. Spectral leakage can be avoided by using periodic time-domain data, which is not always practical. The advantages with frequency-domain identification methods are the option to enhance signal to noise ratio (SNR), its data reduction nature and the possibility to merge several experiments. Another advantage is that an excitation signal applied at time $t=-\infty$ would guarantee the system to be at steady state, thus enabling the user to discard initial conditions [26]. On the other hand, most time-domain methods assume zero initial conditions [21].

\subsubsection{Parametric time-domain methods}

The primary method for system identification in time domain is the prediction error method (PEM). The aim of PEM, like any other identification method, is to find the best estimate of the unknown parameter vector $\theta$ from the measurement data set $Z^{N}=$ $\{u(1), y(1), \ldots, u(N), y(N)\}$, for a chosen model structure $\mathcal{M}(\theta)$.

The main idea of PEM is minimization of prediction errors,

$$
\epsilon(t, \theta)=y(t)-\hat{y}(t \mid \theta), t=1,2, \ldots, N
$$

where $\hat{y}(t \mid \theta)$ is the prediction of $y(t)$ based on the data $Z^{t-1}$.

The discrete-time version of (3.1) is

$$
y(t)=G(q, \theta) u(t)+H(q, \theta) e(t)
$$

with the differential operator $p$ is replaced with the difference operator $q$.

The standard predictor of the system represented by (3.6) is

$$
\hat{y}(t \mid \theta)=H^{-1}(q, \theta) G(q, \theta) u(t)+\left(1-H^{-1}(q, \theta)\right) y(t)
$$

The quadratic criterion is commonly used for the minimization of prediction errors, which yields the estimated parameter vector as follows.

$$
\begin{aligned}
& \hat{\theta}_{N}=\underset{\theta}{\operatorname{minimize}} V_{N}\left(\theta, Z^{N}\right), \\
& V_{N}\left(\theta, Z^{N}\right)=\frac{1}{N} \sum_{t=1}^{N} \frac{1}{2} \epsilon^{2}(t, \theta)
\end{aligned}
$$


In general the optimization is nonlinear and requires numerical search process such as Gauss-Newton algorithm. Good initial values of $\theta$ will be required to avoid local minima. A complete account of PEM methods and its properties can be found in [21].

Subspace methods are popular for state-space models, especially multivariable ones [21]. It estimates the matrices of the state-space model by solving a series of least-squares problems. For this reason subspace methods are computationally efficient compared to PEM. It is also numerically reliable and does not depend on any iterative methods [21]. It does not have any convergence issues or local minimum problem. Subspace methods in general produce good quality estimates [21], though PEM may be optimal in term of model fit to the data. Subspace method does not require any initial values for the parameter, and therefore can be used as a preliminary stage to PEM method.

\subsubsection{Nonparametric frequency-domain methods}

Nonparametric models are almost always in the form of FRFs. Apart from providing valuable information about the underlying system, they could also be an intermediate step in the frequency-domain identification process. Nonparametric methods transform time-domain data into frequency-domain FRFs. They are represented as a table or an array containing complex valued numbers at a large number of frequency points, and are often plotted in a Bode-diagram.

The basic nonparametric estimator is the empirical transfer function estimate (ETFE), defined as

$$
\widehat{G}\left(e^{j \omega_{k}}\right)=\frac{Y\left(\omega_{k}\right)}{U\left(\omega_{k}\right)}
$$

where,

$$
\begin{aligned}
& Y\left(\omega_{k}\right)=\frac{1}{\sqrt{N}} \sum_{n=1}^{N} y(n) e^{-j \omega_{k} n} \\
& U\left(\omega_{k}\right)=\frac{1}{\sqrt{N}} \sum_{n=1}^{N} u(n) e^{-j \omega_{k} n}
\end{aligned}
$$

are the DFTs of time-domain discrete-time signals $y(n)$ (output) and $u(n)$ (input), respectively. Here $\omega_{k}=k \frac{2 \pi}{N}, k=1,2, \ldots, N$ are discrete frequencies and $N$ is the number of time samples. The question is how different is the quantity $\widehat{G}\left(e^{j \omega_{k}}\right)$ from its continuous time equivalent $G\left(j \omega_{k}\right)$. It turns out that the agreement between the two is good enough for frequencies less than one tenth of the sampling frequency [13]. 
ETFE is unbiased and its variance approaches zero as $N$ tends to infinity, as long as the time-domain data is periodic with integer number of periods. If not periodic, it will be asymptotically unbiased but the variance approaches the signal to noise ratio (SNR) [21].

Some other estimators are the $H 1$ estimator, the arithmetic mean (ARI) estimator, the joint input-output (JIO) estimator and the errors-in-variable (EIV) estimator. These estimators are studied in [27] and [12].

\subsubsection{Parametric frequency-domain methods}

The system can be represented in the frequency domain as

$$
Y\left(\omega_{k}\right)=G\left(e^{j \omega_{k}}, \theta\right) U\left(\omega_{k}\right)+H\left(e^{j \omega_{k}}, \theta\right) E\left(\omega_{k}\right)
$$

The frequency-domain data set may be available as the DFT of the input and output signals, $U\left(\omega_{k}\right)$ and $Y\left(\omega_{k}\right)$ respectively, but also as the ETFE, $\hat{G}\left(e^{j \omega_{k}}\right)$.

The most common criterion for parametric identification, the weighted least square criterion, is

$$
\underset{\theta}{\operatorname{minimize}} V_{N}\left(\theta, Z^{N}\right)
$$

where

$$
V_{N}\left(\theta, Z^{N}\right)=\sum_{k=1}^{N}\left|Y\left(\omega_{k}\right)-G\left(e^{j \omega_{k}}, \theta\right) U\left(\omega_{k}\right)\right|^{2} W_{k}
$$

for data set $Z^{N}=\left\{U\left(\omega_{1}\right), Y\left(\omega_{1}\right), \ldots, U\left(\omega_{N}\right), Y\left(\omega_{N}\right)\right\}, N$ being the number of discrete frequencies of the DFT, and

$$
V_{N}\left(\theta, Z^{N}\right)=\sum_{k=1}^{N}\left|\widehat{G}\left(e^{j \omega_{k}}\right)-G\left(e^{j \omega_{k}}, \theta\right)\right|^{2} W_{k}
$$

for data set $Z^{N}=\left\{\widehat{G}\left(e^{j \omega_{1}}\right), \hat{G}\left(e^{j \omega_{2}}\right), \ldots, \widehat{G}\left(e^{j \omega_{N}}\right)\right\}$.

The weighting function $W_{k}$ can be based on the noise spectrum. This criterion, in the frequency domain, has duality with the PEM in the time domain [21].

The resulting optimization problem is its nonlinear in parameters and consequently it requires a numeric search algorithm. It also requires initial values for the parameter vector in order to avoid local minima. 
Subspace methods are available for frequency domain also. Some references are [28] and [29].

\subsubsection{Closed-loop identification}

Some systems have to be identified while they are operated in a stabilizing closed loop. This could be due to the inherent instability of the open-loop plant or the process cannot be stopped for economic or safety reasons. Figure 3.2 shows a system in closed loop. $G_{0}$ is the system plant and $F_{y}$ is the controller. Closed-loop data in general contains less information about the system (closed loop is meant to suppress certain behavior) [21]. But the major problem is the correlation of noise signal $v$ with the input signal $u$, due to feedback. This may cause standard nonparametric methods to produce biased estimates [21]. However, PEM works fine as long as the true system is contained in the model set (or model structure) [21]. For closed loop systems, the reference input $r$ is considered as the system input rather than $u$. The excitation signal is applied to $r$.

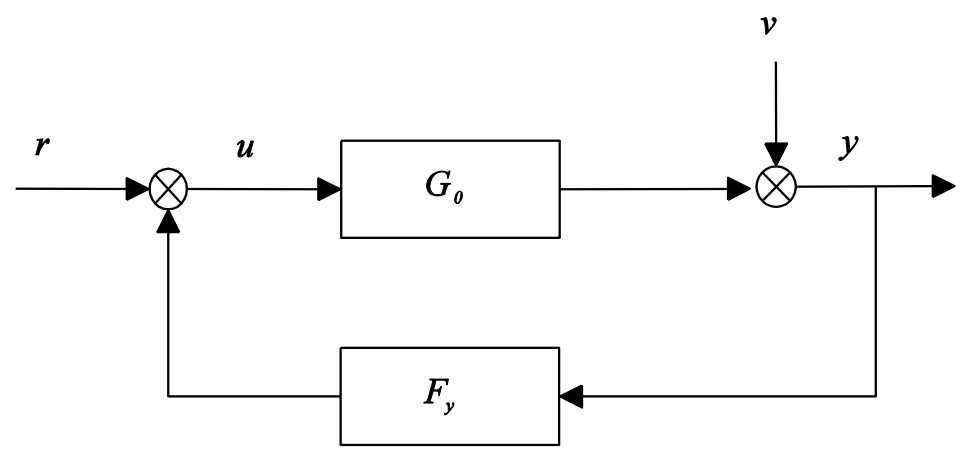

Figure 3.2. A closed loop system

According to Ljung (1999) [21], there are three standard methods to identify closedloop systems.

Direct method: Ignore the feedback and apply standard methods.

Indirect method: Identify the system as an open loop system with the closed-loop transfer function from $r$ to $y$. Then compute the open-loop model $G_{0}$ from the identified model (algebraically) using the model of the controller $F_{y}$. Of course this is possible only if the controller model $F_{y}$ is known (and linear).

Joint input-output method: Consider the system as MIMO with inputs $r$ and $v$, and outputs $u$ and $y$. Identify such a model after choosing a parameterized model structure for both $G_{0}$ and $F_{y}$.

Standard identification methods can be used in all three cases. The direct method with PEM should be considered as the primary method [21]. A comprehensive analysis of closedloop identification can be found in [30]. 


\subsection{Experiment design}

The quality of identification will be as good as the input-output data set used in the process. It is important to generate informative data by performing an appropriately designed experiment. The design of excitation signal involves choosing the frequencies, amplitudes, sampling rates, and so on.

\subsubsection{Input spectrum}

The spectrum of the excitation signal is important because it influences the bias and variance of the estimated model. For example, the bias and variance of ETFE for an open-loop system is as shown below [21].

$$
\begin{gathered}
E\left(\widehat{G}_{N}\left(e^{j \omega T_{S}}\right)\right)=G_{0}\left(e^{j \omega T_{S}}\right)+\frac{\rho(N)}{U_{N}(\omega)} \\
\operatorname{Cov}\left(\widehat{G}_{N}\left(e^{j \omega T_{s}}\right)\right)=\frac{1}{\left|U_{N}(\omega)\right|^{2}}\left(\Phi_{v}(\omega)+\rho_{2}(N)\right)
\end{gathered}
$$

where $\left|\rho_{1}\right| \leq C_{1} / \sqrt{N}$ and $\left|\rho_{2}\right| \leq C_{2} / N$ for some constants $C_{1}$ and $C_{2}$, and $G_{0}$ is the true system. Both the expectation and covariance depends on $U_{N}(\omega)$. The excitation can be designed to achieve a desired accuracy based on some criterion, for example the relative accuracy [13],

$$
\Phi_{u}(\omega)=\text { const } \cdot \frac{\Phi_{v}(\omega)}{\left|G_{0}\left(e^{j \omega T_{s}}\right)\right|^{2}}
$$

where $\Phi_{u}(\omega)$ and $\Phi_{v}(\omega)$ are the spectrum of input and noise respectively. $\Phi_{u}(\omega)$ should be such that more power is injected into the system at frequencies which are more informative, for example, around the expected poles of the system. It could also be limited by practical constraints such as the maximum limit for the amplitude of $u$.

\subsubsection{Periodicity of input}

Periodic inputs have number of advantages. For nonparametric method such as ETFE, which relies on DFT of signals, periodic signals with integer number of periods can significantly improve the variance of the estimate. For periodic signals, ETFE is unbiased and its variance decays as $1 / N$ [21]. If the data is non-periodic, ETFE will be asymptotically unbiased and the variance will be the same as its SNR [21].

\subsubsection{Selection of excitation signal}

Once the desired spectrum is known the signal could be designed. A straightforward approach is to use stepped sine excitation, where the system is excited with a sequence of frequencies, one after the other. This is time consuming and is less preferred to other excitation strategies. However, stepped sine excitation would have the best SNR. The frequency points can be 
spaced linearly or logarithmically. It may also be made denser around expected poles of the system.

Other more commonly used excitation signals are multisine, filtered white noise, swept sine, and pseudo-random binary sequence (PRBS). Most of them contain multiple frequencies at a time, so that all frequencies of interest are applied to the system at once. More details can be found in [21] and [23].

\subsection{Model validation}

System identification is not complete without model validation. In this section some common technique that are used for validating an identified model are introduced. The methods are described in detail in [21].

\subsubsection{Cross-validation}

Cross-validation is validation through simulation. The model is validated against an independent data set of the true system. It could be done in either time domain or frequency domain. In the case of time domain, the time-domain outputs of the model and the true system, when excited with the same input, are compared. For frequency-domain validation, the frequency response function (bode plot) of the model and true system are compared and quantified with a well-defined fit. More details can be found in [21].

\subsubsection{Residual analysis}

The basic idea of residual analysis is as follows. If the identification has been done properly, the residuals, or the error between true system response and model response, will be a completely random signal or white noise. Residuals are the perdition errors in (3.5). White residuals indicate two things; that the method has done a good job in extracting all the information present in the response data, and also that the true model was contained in the model structure.

There are two tests to analyze the residuals [21]. One is the whiteness test where whiteness of the residuals at each output is tested. This can be done by inspecting the autocorrelation function (time-domain) of the residuals or its spectrum (frequency-domain). The second test is called the independence test, where the correlation between the residuals and the inputs are checked. A good model has residuals that are uncorrelated to past inputs.

\subsubsection{Model uncertainty}

The quality of the estimated model can be measured by its bias and variance. Correlation of noise to the input signals usually introduces bias in to the system. Variance is usually impacted by signal to noise (SNR) ratio of the input-output signals. Identification methods could calculate the covariance matrix for the parameter vector in the model. This information can be used to plot confidence intervals in various plots, such as the pole-zero map, bode plot etc. This is a good way to visualize the uncertainty in the model. 


\section{Chapter 4}

\section{Identification of the AMB}

\section{system}

In this chapter, the problem for identification of AMB is formulated with specific details. Some important issues with regard to identification of AMB are discussed and crucial decisions are taken. Based on these decisions, a procedure for identification for commissioning is developed.

\subsection{Problem specification}

\subsubsection{Requirements}

The requirements for identification for commissioning were presented in Sec.1.3.2. Here they are discussed in more detail.

\section{Identify an accurate system model}

A linear state-space model, possibly of the model structures presented in Sec. 2.7 is the aim of identification. The AMB to be identified is to be considered as a flexible rotor with two flexible modes. The relevant model structure of the complete plant is Eqn. (2.13).

\section{Identify a set of special system parameters}

There are two sets of parameters considered to be relevant for verification and diagnostics. One is the stiffness parameters defined in (2.3), and the other is the rotor parameters, namely its eigenfrequency and damping ratio for each flexible mode.

\section{The process should be robust against noise}

Signal to noise ratio is crucial for the accuracy of the model estimate. The commissioning site is assumed to be noisy. The procedure used has to be based on methods which improves SNR.

4. The procedure should be independent of the FEM

PEM methods require initial models for their underlying nonlinear optimization routines. If it comes to that, nominal models for flexible-rotor model (Sec. 2.6), which can only be generated through a process of FEM, cannot be used in the procedure.

5. The procedure should be automated as well as possible

The primary challenge seems to be the automation of excitation experiment. It could be done based on the principles discussed in Sec. 3.4.

\subsubsection{Prerequisites}


- The rotor has to be levitated and stabilized using a preliminary feedback controller.

- System identification toolbox (IDENT) of MATLAB.

\subsection{Issues in identification of an AMB system}

In this section, various issues and challenges pertaining to system identification of AMB are discussed and crucial decisions are taken, with an eye on the requirements for commissioning.

\subsubsection{Time-domain vs. Frequency-domain identification}

Identification in the frequency domain appears to be the primary choice when dealing with resonant mechanical systems and vibration analysis. Some advantages of frequency domain identification were outlined in Sec. 3.3.1. In addition to that, it also has advantages in filtering (improve SNR) and for estimating the noise spectrum. For these reasons, frequency domain identification is selected for identification for commissioning. Identification in frequency domain is time consuming, but it is still preferable to relatively lower SNR of time-domain identification (compared to stepped sine excitation, for example). Frequency-domain identification is done in two steps. First, a nonparametric model (basically the frequency response data), of the open-loop plant, is identified. Second, a suitable identification method fits this data to a selected model structure.

\subsubsection{Closed loop identification}

An unstable system, such as an AMB system, can only be identified in closed loop. The basic principles were introduced in Sec. 3.3.5. Since frequency domain identification has been chosen, the closed-loop identification is going to be a nonparametric method. The standard method of ETFE is known to produce biased estimates, but can be acceptable if the SNR is large. According to Ljung [21], the ETFE estimate for closed loop (see Fig. 3.2) tends to

$$
\widehat{\mathrm{G}}\left(e^{j \omega T_{s}}\right)=\frac{G_{0}\left(e^{j \omega T_{s}}\right) \Phi_{r}(\omega)-F_{y}\left(e^{-j \omega T_{s}}\right) \Phi_{v}(\omega)}{\Phi_{r}(\omega)+\left|F_{y}\left(e^{j \omega T} s\right)\right|^{2} \Phi_{v}(\omega)} .
$$

For cases of large SNR, i.e. large $\left|\Phi_{r}(\omega)\right|$ compared to $\left|\Phi_{v}(\omega)\right|, \widehat{G}\left(e^{j \omega T_{s}}\right)$ tends to $G_{0}\left(e^{j \omega T_{s}}\right)$ which is the true model.

ETFE for MIMO systems have the form,

$$
\widehat{G}_{0}\left(e^{j \omega}\right)=Y_{N}(\omega) U_{N}(\omega)^{-1}
$$

where 


$$
\begin{gathered}
U_{N}\left(\omega_{k}\right)=\left[\begin{array}{cccc}
U_{1}^{(1)}\left(\omega_{k}\right) & U_{1}^{(2)}\left(\omega_{k}\right) & \ldots & U_{1}^{\left(N_{e}\right)}\left(\omega_{k}\right) \\
U_{2}^{(1)}\left(\omega_{k}\right) & U_{2}^{(2)}\left(\omega_{k}\right) & \ldots & U_{2}^{\left(N_{e}\right)}\left(\omega_{k}\right) \\
\vdots & \vdots & & \vdots \\
U_{N_{u}}^{(1)}\left(\omega_{k}\right) & U_{N_{u}}^{(2)}\left(\omega_{k}\right) & \ldots & U_{N_{u}}^{\left(N_{e}\right)}\left(\omega_{k}\right)
\end{array}\right] . \\
Y_{N}\left(\omega_{k}\right)=\left[\begin{array}{cccc}
Y_{1}^{(1)}\left(\omega_{k}\right) & Y_{1}^{(2)}\left(\omega_{k}\right) & \ldots & Y_{1}^{\left(N_{e}\right)}\left(\omega_{k}\right) \\
Y_{2}^{(1)}\left(\omega_{k}\right) & Y_{2}^{(2)}\left(\omega_{k}\right) & \ldots & Y_{2}^{\left(N_{e}\right)}\left(\omega_{k}\right) \\
\vdots & \vdots & & \vdots \\
Y_{N_{y}}^{(1)}\left(\omega_{k}\right) & Y_{N_{y}}^{(2)}\left(\omega_{k}\right) & \ldots & Y_{N_{y}}^{\left(N_{e}\right)}\left(\omega_{k}\right)
\end{array}\right] .
\end{gathered}
$$

$N_{u}$ : number of inputs of MIMO system

$N_{y}$ : number of outputs of MIMO system

$N_{e}$ : number of experiments, $N_{e} \geq N_{u}$

$Y_{N}(\omega)$ and $U_{N}(\omega)$ are the discrete Fourier transform (DFT) of individual outputs and inputs of the MIMO system. As indicated above, as many independent experiments are required as there are inputs to the system. This is a specialty of MIMO systems. Full analysis and discussions are presented in [23].

\subsubsection{Excitation experiment}

The general principles of experiment design were presented in Sec. 3.4. The modern approach to excitation for identification is broadband signals, either a multisine, band limited white noise or pseudorandom binary sequence (PRBS) [21]. Traditional methods are based on stepped sine or swept sine signals, in which the frequency is varied in discrete steps across the bandwidth of the system. Broadband signals are efficient in the sense that a single experiment would contain all the required frequencies. But stepped sine excitation is chosen for the following reasons. It has very high SNR, because the full power of the signal is concentrated at one frequency a time. It will always generate informative data since a large number of frequencies are included in the experiment. From the discussion for closed-loop identification, it is obvious that a high SNR is crucial for obtaining unbiased ETFE.

\subsubsection{Model structures}

The most important aspect of system identification is the choice of model structures. In the case of AMB, which is a mechanical system, analytical modeling is an acceptable way to finalize a particular model structure, even without testing it. Mainly because the motivation for such a model structure is very strong from the point of view of physics, and the user would want to enforce such a model structure to the data. Such an approach would ignore unexpected dynamics, and therefore, caution is advised here. If the user has sufficient confidence in the analytical model, this a good way forward. In this thesis, such an approach is adopted, and the model structures developed in Chapter 2 will be used.

\subsubsection{Identification methods}


Frequency domain identification has two parts, namely: nonparametric and parametric identification. ETFE has been chosen for nonparametric identification. The output of the nonparametric step is the frequency response data (or Bode plot) of the open loop (unstable) plant. This constitutes the data set for the frequency-domain parametric identification step. The primary method for frequency (or time) domain parametric identification is PEM [21]. However, for MIMO state-space model structures, subspace methods are convenient and widely used. The system identification toolbox (IDENT) in MATLAB supports both PEM and subspace methods in the frequency domain.

The basic principles of PEM and subspace methods can be found in [21]. Subspace method can at best identify models in canonical forms, but PEM has no such limitations. PEM supports identification of models in any arbitrary form but requires an initial model. The standard implementation of PEM in IDENT includes a preliminary subspace stage. This relieves the user from providing initial model parameters, but the form of the model is limited to standard canonical forms or fully free parameterization of all elements of the state-space matrices. IDENT also supports grey-box identification, which is based on PEM, to identify an arbitrarily parameterized grey-box model, but this would require an initial model parameter. This method does not have a preliminary subspace stage. PEM and subspace identification methods are available in IDENT as ssest ( ) and n4sid () routine calls [31]. It is important to remember that ssest () first calls n4sid () and then computes the actual PEM on the preliminary results obtained from $\mathrm{n} 4 \mathrm{sid}()$. Therefore, ssest () will almost always give a refined version of $n 4 \mathrm{sid}()$.

To sum up, the options available in IDENT are:

1. Subspace (n4sid ()): Requires the model order and the form (canonical or free) of the state-space model from the user. Automatically identifies the modes of the system.

2. PEM(ssest ()): This can only refine the model estimate from n4sid (). Nothing more. Therefore, ssest () could be preferred over n 4 sid (), almost everywhere.

3. Structured PEM (grey-box): Can identify model structures in any arbitrary form, not limited to the canonical forms. The state-space matrices can be parameterized with any arbitrary set of parameters, and those parameters can be estimated in addition to the model. The downside is that it requires an initial value for the parameter vector, from the user. n 4 sid ( ) cannot estimate an initial model in arbitrary form.

It has to be noted that IDENT allows for the identification of only output-error model structures in the frequency domain [31]. Output-error models structures are models with noise model equal to unity.

\subsection{Strategy for identification}

The objective is to identify a flexible-rotor model with a model structure as given in Eqn. (2.13). The nonparametric model will be identified for the complete plant. The main choices are between ssest () and grey-box methods. The complete plant could be identified using 
ssest (), but that model could at best be of canonical form. Such a model can be used for controller synthesis, but will not be able to estimate the required parameters. On the other hand, grey-box will be able to estimate the parameters but will require an initial model. An initial model for a flexible-rotor AMB can be generated by using an FEM. This dilemma can be addressed by separate identification of rigid and flexible modes of the system. Another issue is that the amplifier modes may not be accurately identified if it is modeled as a part of the complete model.

\subsubsection{Separate identification of rigid and flexible modes}

The FRF is expected to have a resonance peak for each flexible mode in the system. These peaks are situated in the higher frequency region. This is because the rotor is a highly resonant structure with very low internal damping, and the modes representing the flexible eigenmodes will be of higher frequency. This is a known characteristic of flexible rotor and can be observed in the FEM model. This region of FRF, which contains all the resonance peaks, can be considered as the flexible region. The remaining low frequency region will contain the two rigid modes of the rotor and also the amplifier modes. This region can be considered as the rigid region. Section 2.5 explains what the two rigid modes are. The rigid and flexible regions can be easily distinguished by the user, once the nonparametric model is identified (see Fig. $5.6)$.

The above observation leads to the following idea. If the FRF is split into two regions, the rigid region can be used to identify the rigid-rotor model (2.12), the flexible region can be used to identify only the flexible modes, and the complete plant model can be obtained by combining the two models. This idea is interesting because it allows the rigid-rotor model to be identified using grey-box method and the flexible modes to be identified by the ssest () method. The analytical model of (2.12) can be used as the initial model for the grey-box identification while the flexible modes can be identified in a standard canonical form. The greybox model can be parameterized in terms of the stiffness constant, provided all other physical parameters in model (2.12) are known. Furthermore, the rotor parameters can be extracted from the identified flexible modes if it is expressed in modal canonical form (see (4.8)). This solves the original dilemma and allows the identification procedure to identify all the required parameters as well as be independent of the FEM model. The splitting of the complete plant model will not affect the accuracy of estimation since the informative parts of rigid and flexible regions are far apart in the FRF.

\subsubsection{Independent identification of amplifier}

Independent identification of the amplifier modes is possible if the coil currents in the electromagnets are measured. Independent identification is interesting because this will allow for the more accurate identification of the modes. The only price to pay is the sampling of an additional signal, the coil current, during the excitation experiment. This is certainly acceptable.

\subsection{Identification of AMB systems: A procedure}


In this section a procedure for identification of AMB systems for commissioning is presented. The procedure is developed based on the decisions taken in Sec. 4.2 and the strategy outlined in Sec. 4.3. The procedure is strictly for a single plane ( $x$-plane) of AMB system. It may be repeated to identify the other plane ( $y$-plane).

\subsubsection{Step 1: Identification experiment}

The first step in an identification procedure is to design and perform an experiment. Stepped sine has been chosen as the excitation approach. The system is excited after the rotor has been levitated. Since the system under consideration is $2 \times 2 \mathrm{MIMO}$, at least two experiments are required. This is in line with the theory discussed in Sec. 4.2.2. For identification of AMB in $x$-plane, $N_{u}=N_{y}=2$. One experiment is done by exciting the system through the first input $r_{A}$ only, and the second is done by exciting the second input $r_{B}$ only.

The design of stepped sine is the same for both experiments. The amplitude profile is generated according to (3.18), but subject to the limits imposed by the linear region of AMB. The noise spectrum required in (3.18) is estimated in a preliminary step where an integer number of periods of output signal (for a single arbitrary frequency) is averaged and then subtracted from the original signal. More power is injected at around rigid poles of the system and not the flexible modes, since the latter would lead to excitation of eigenfrequencies. The frequency grid is logarithmically spaced but with denser regions around the flexible poles. The frequency range is selected to include the required number of eigenmodes and also the amplifier bandwidth.

Once the design parameters are determined, the excitation process is automated. During the experiment signals $\mathrm{u}, \mathrm{i}$ and $\mathrm{y}$ are sampled with a sampling rate which is at least ten times the bandwidth of the system. An integer number of periods of signals are recorded and used for future ETFE computation. This is important to reduce the variance of the estimate.

\subsubsection{Step 2: Generation of the nonparametric model}

Since it has been decided that the amplifier has to be identified independently, two nonparametric models, one for the amplifier and the other for the complete plant, are required for further steps of the procedure.

For the $2 \times 2$ MIMO AMB system, ETFE is defined as:

$$
\begin{gathered}
\hat{G}\left(e^{i \omega_{k}}\right)=Y_{N}\left(\omega_{k}\right) U_{N}\left(\omega_{k}\right)^{-1} . \\
U_{N}\left(\omega_{k}\right)=\left[\begin{array}{ll}
U_{A}^{(A)}\left(\omega_{k}\right) & U_{A}^{(B)}\left(\omega_{k}\right) \\
U_{B}^{(A)}\left(\omega_{k}\right) & U_{B}^{(B)}\left(\omega_{k}\right)
\end{array}\right], Y_{N}\left(\omega_{k}\right)=\left[\begin{array}{cc}
Y_{A}^{(A)}\left(\omega_{k}\right) & Y_{A}^{(B)}\left(\omega_{k}\right) \\
Y_{B}^{(A)}\left(\omega_{k}\right) & Y_{B}^{(B)}\left(\omega_{k}\right)
\end{array}\right] .
\end{gathered}
$$

where the subscript corresponds to the input/output, and the superscript corresponds to different experiments. For example, $U_{B}^{(A)}(\omega)$ means the DFT of input $u_{B}$ in experiment A. While considering the $x$-plane of AMB, the system has two inputs $\left(u_{A}, u_{B}\right)$ and two outputs $\left(y_{A}, y_{B}\right)$ 
$(2 \times 2 \mathrm{MIMO})$. In such cases a minimum of two experiments are required. Excitation applied to $r_{A} / r_{B}$, reference signal at side $\mathrm{A} / \mathrm{B}$, constitutes experiment $\mathrm{A} / \mathrm{B}$. After obtaining DFT of $u$ or $y$, only one frequency component is considered, the one which is used for excitation. This further isolates the model from noise.

The two amplifier modes are decoupled and can be identified as two independent SISO systems. ETFE for the amplifiers are obtained by

$$
\begin{aligned}
& \hat{G}_{a A}\left(e^{i \omega_{k}}\right)=I_{A}^{(A)}\left(\omega_{k}\right) / U_{A}^{(A)}\left(\omega_{k}\right) \\
& \hat{G}_{a B}\left(e^{i \omega_{k}}\right)=I_{A}^{(A)}\left(\omega_{k}\right) / U_{B}^{(B)}\left(\omega_{k}\right)
\end{aligned}
$$

where the subscripts and superscripts are defined in a similar way, and $\widehat{G}_{a A}$ and $\widehat{G}_{a A}$ are models for amplifier A and B respectively.

\subsubsection{Step 3: Identification of the amplifier model}

The two independent amplifiers (A/B) are identified with ssest () using their respective nonparametric model obtained in the previous step. ssest ( ) is configured to output a model in modal canonical form. They are then combined to form a $2 \times 2$ MIMO system to match the analytical model of (2.2).

\subsubsection{Step 4: Identification of the rigid-rotor model and the AMB stiffness constants}

The nonparametric model of the complete plant, which was obtained in Step 2, is split into rigid and flexible regions. The frequency at which this split is made is based on intuition. The split should be right before the influences of the flexible modes are seen in the FRF.

The rigid region becomes the frequency-domain data set for the identification of the rigid-rotor model. Identification of rigid-rotor model is done with grey-box method. A greybox model is set up in IDENT using the model in (2.12) and parameterized with only the stiffness constants. Other parameters in model (2.12) are assumed to be available at the time of commissioning. The parameter set $\left\{k_{S A}, k_{S B}, k_{i A}, k_{i B}\right\}$ is the unknown parameter vector in the grey-box setting. The grey-box method estimates the parameter vector through an internal optimization process. The nominal values of the parameters are used as the initial parameter vector. The stiffness constants have to be proven to be structurally identifiable. This can be done with similarity transformation approach [24]. The proof can be found in Appendix B.

This step outputs estimates for the rigid-rotor model as well as individual values for the AMB stiffness constants.

\subsubsection{Step 5: Identification of the flexible modes and the rotor parameters}

With the rigid modes of the system identified, what remain are the flexible modes. Since flexible modes of the rotor structure are expected to be highly resonant (prominent features in 
the nonparametric model), there is little risk for the preliminary subspace method to identify spurious modes. Therefore, ssest ( ) is used to fit the flexible region (resonance peaks) of the nonparametric model to a state-space model in modal form.

The resulting system matrix contains one pair of complex conjugate poles, $-\sigma_{f} \pm i \omega_{d f}$, for each flexible mode present in the data, along its main diagonal. The flexible-rotor parameters, the undamped natural frequency $\left(\omega_{n}\right)$ and the damping ratio $(\zeta)$, are obtained from the complex conjugate pole, which consists of damped natural frequency $\left(\omega_{d f}\right)$ and attenuation $\left(\sigma_{f}\right)$, using the well-known equation

$$
\sigma_{f}=\zeta \omega_{n}, \quad \omega_{d f}=\omega_{n} \sqrt{1-\zeta^{2}}
$$

\subsubsection{Step 6: Combine the rigid and flexible modes}

In this step the rigid model obtained in Step 4 is diagonalized and then combined to the flexible mode model obtained in Step 5, which is already in modal form. This combination is just a matter of stacking the state-space matrices together, since, in modal form, all modes (rigid or flexible) are decoupled from each other. Thus the complete plant model, in state-space form, is obtained in this step.

In modal canonical form, the eigenvalues (poles) of the system appear in the main diagonal of the system matrix. For example, for a system with one flexible mode for the rotor, it would look like,

$$
\left[\begin{array}{cccccccc}
-\omega_{l A} & 0 & 0 & 0 & 0 & 0 & 0 & 0 \\
0 & -\omega_{l B} & 0 & 0 & 0 & 0 & 0 & 0 \\
0 & 0 & -\sigma_{f} & \omega_{d f} & 0 & 0 & 0 & 0 \\
0 & 0 & -\omega_{d f} & -\sigma_{f} & 0 & 0 & 0 & 0 \\
0 & 0 & 0 & 0 & \omega_{\mathrm{r} 2} & 0 & 0 & 0 \\
0 & 0 & 0 & 0 & 0 & -\omega_{\mathrm{r} 2} & 0 & 0 \\
0 & 0 & 0 & 0 & 0 & 0 & \omega_{\mathrm{r} 1} & 0 \\
0 & 0 & 0 & 0 & 0 & 0 & 0 & -\omega_{\mathrm{r} 1}
\end{array}\right]
$$

where,

$$
\begin{aligned}
& \omega_{l A}, \omega_{l B}: \text { are amplifier bandwidths of AMB A, B. } \\
& \sigma_{f}, \omega_{d f}: \text { attenuation and damped natural frequency of the flexible mode. } \\
& \omega_{\mathrm{r} 1}, \omega_{\mathrm{r} 2}: \text { corner frequencies of the two rigid modes. }
\end{aligned}
$$

The system matrix in (4.9) is the diagonalized version of the one in (2.13). 


\section{Chapter 5}

\section{Experimental Results}

The procedure which has been developed in the preceding chapter was tested on a prototype AMB system. In this chapter the prototype system as wells as the experiment carried out is described in detail. The intermediate results are presented in the same order of steps of the procedure. Finally the identified models are validated.

\subsection{Test system}

The test system can be summarized by the block diagram in Fig. 5.1. It consists of a nonrotating rotor supported by two AMBs at opposite ends. The two sides are referred to as A and B. The motor is situated between the two AMBs. No load was attached to the rotor during the experiment. The plant, as defined earlier, is a combination of the amplifier, the two AMBs and the rotor. The main controller, which is a dSPACE based system, controls the positions of the rotor ends. The displacements of the rotor are measured by eddy-current sensors and fed back to the controller. Table 5-1 lists some parameters of the test system.

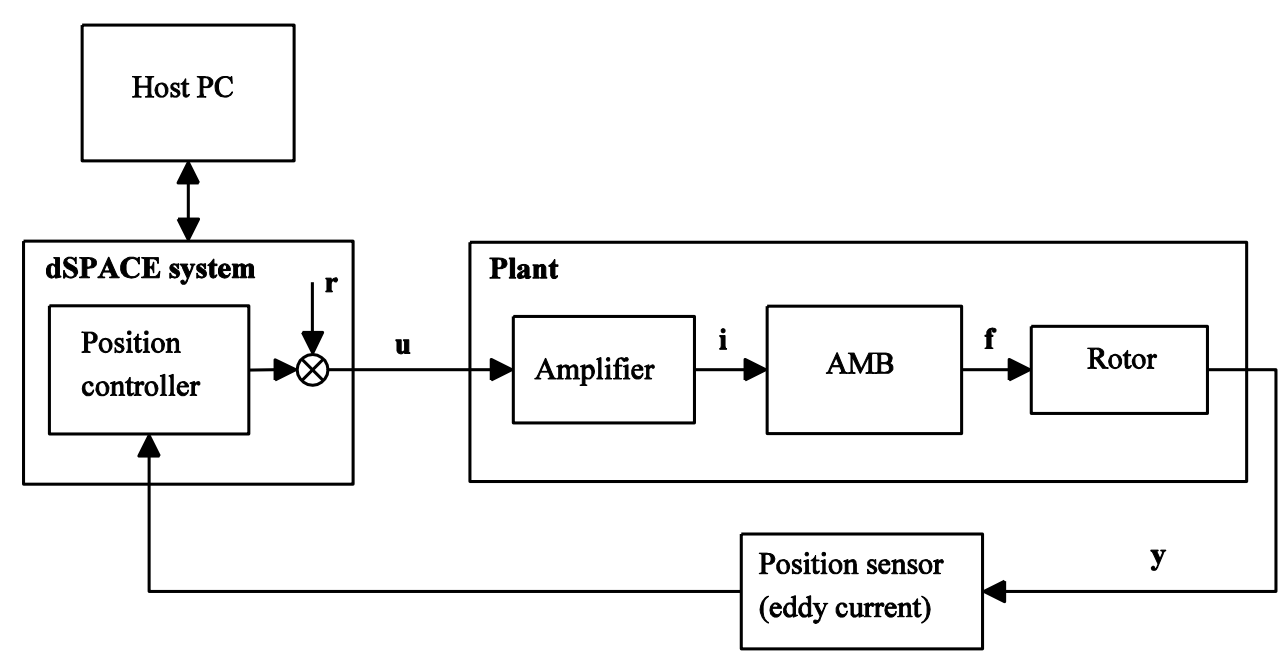

Figure 5.1 Test system block diagram. Signals $\mathbf{r}, \mathbf{u}, \mathbf{i}, \mathbf{f}$ and $\mathbf{y}$ represents reference, control current, coil current, force and output displacement signals, respectively.

\subsubsection{Position controller}

A decentralized proportional-integral-derivative (PID) control algorithm is implemented in the dSPACE controller hardware unit. This is a preliminary controller which accomplishes satisfactory levitation for the purpose of the experiment. The controller outputs the desired control current signal ' $u$ ' as per the control law. The user can program and automate the 
experiment and then insert the excitation signal into the signal represented by ' $r$ '. The experimental data, i.e. the signals $\mathrm{u}, \mathrm{i}$ and $\mathrm{y}$, are sampled and sent to the host PC.

\subsubsection{Host PC}

This is a general purpose computer which runs a suitable application program by which the dSPACE controlled is programmed / monitored. It also collects the experimental data sent by the dSPACE controller. The current signals $r, u$ and i are represented in units of Ampere (A), while the displacement signal $\mathrm{y}$ is in units of meter $(\mathrm{m})$. The identification procedure, implemented in MATLAB, operates on this data and outputs the required plant model and its associated parameters.

\subsubsection{AMB}

Two AMBs support the rotor at its opposite ends. In each plane ( $x$ or $y$ ), each AMB (A or B) consists of a pair of electromagnets configured in differential driving mode, as described in Sec. 2.1. The position controller generates one control signal for each differential pair, resulting in a total of four current control signals. The AMBs are characterized by two stiffness constants, as shown in (2.3). The nominal values of these constants are included in Table 5-1.

\subsubsection{Amplifier}

Every electromagnet is energized by an amplifier unit. The amplifier block in Fig. 5.1 is in fact a closed-loop system by itself. Its design is shown in Fig. 5.2. There are as many independent such units as there are electromagnets. Consequently there are eight amplifier units in total. These units are custom made.

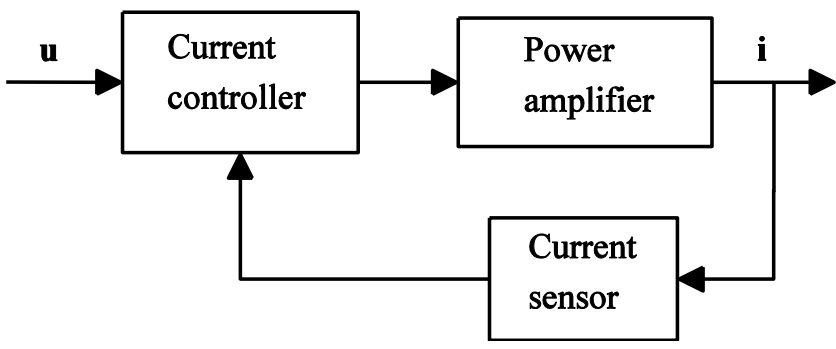

Figure 5.2 Amplifier system (closed loop)

\subsubsection{Rotor}

A flexible rotor with up to two significant flexible modes is used in the experiment. Fig. 5.3 shows the approximate structure of the rotor. It is nearly symmetric except for a small feature at side B. The various dimensions and physical parameters of the rotor are included in Table $5-1$. The moment of inertia (MOI) and center of gravity (COG) of the rotor are assumed to be known. 


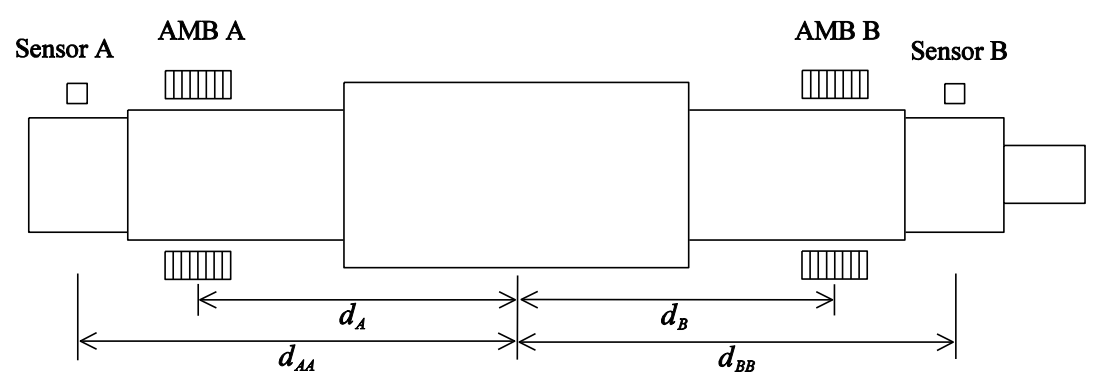

Figure 5.3 Rotor structure and dimensions. Bearings A and B are separated from the center of gravity of the rotor by $\boldsymbol{d}_{\boldsymbol{A}}$ and $\boldsymbol{d}_{\boldsymbol{B}}$. The sensors are separated from the same by $\boldsymbol{d}_{\boldsymbol{A} \boldsymbol{A}}$ and $\boldsymbol{d}_{\boldsymbol{B} \boldsymbol{B}}$.

\subsubsection{Position sensor}

The displacement of the two rotor ends (A or B) in each plane ( $x$ and $y$ ) are measured using eddy-current sensors. In total there are four such sensors. The dynamics of the sensors are assumed to be fast in comparison to other parts of the system, and hence it is neglected.

Table 5-1 Test system parameters

\begin{tabular}{cccl}
\hline Parameter & Value & Units & \multicolumn{1}{c}{ Description } \\
\hline$m$ & 280 & $\mathrm{Kg}$ & Mass of the rotor \\
$d_{A}$ & 0.418 & $m$ & Distance of AMB A from COG \\
$d_{B}$ & 0.404 & $m$ & Distance of AMB B from COG \\
$d_{S A}$ & 0.484 & $m$ & Distance of Sensor A from COG \\
$d_{S B}$ & 0.470 & $m$ & Distance of Sensor B from COG \\
$I_{r}$ & 10.87 & $K g m^{2}$ & MOI of the rotor \\
$K_{S A} / K_{S B}$ & $2.3 \times 10^{6}$ & $N / m$ & Displacement stiffness of AMB A/B \\
$K_{i A} / K_{i B}$ & 340 & $N / A$ & Current stiffness of AMB A/B \\
$I_{b}$ & 2.75 & $A$ & Bias current of electromagnet \\
$y_{l}$ & \pm 80 & $\mu m$ & Linear region of rotor displacement \\
\hline
\end{tabular}

\subsection{Experiment details}

Stepped sine has been chosen as the excitation method for the identification experiment. The general principles of designing a stepped sine experiment were outlined in Sec. 3.4. In general the excitation signal is applied to the reference signal $r$, and the signals $u, i$, and $y$ are recorded by the controller. It must be pointed out that no fresh experiment was conducted as a part of this thesis because of inaccessibility of the system. But rather a couple of existing experimental data sets were used. Normally a single data set should have all the relevant signals, $\mathrm{u}, \mathrm{i}$, and $\mathrm{y}$. In the absence of such a data set, two separate experimental data sets, one containing the signals $\mathrm{u}$ and $\mathrm{i}$, and the second containing the signals $\mathrm{u}$ and $\mathrm{y}$, where used for identification of the amplifier and the complete plant, respectively. The excitation experiment which generated the two data sets was not designed based on the principles discussed in Sec. 3.4, but were still acceptable. The experiments which generated the two data sets are discussed below. 


\subsubsection{Amplifier data set}

This data set contains the signals $u$ and $i$, meaning the input-output data of the amplifier. The signals $u$ and $i$ are defined in Sec. 2.2. The amplifier model structure is presented in Sec. 2.3. It can be seen from (2.2) that the amplifier model for a plane ( $x$ or $y$ ) is made up of two decoupled SISO systems, thereby facilitating their independent identification. The stepped sine experiment has the following design.

1. The frequency range covers the amplifier bandwidth (10 to $3000 \mathrm{~Hz})$.

2. The frequency grid is in steps of $20 \mathrm{~Hz}$ throughout the range.

3. For frequencies up to $1000 \mathrm{~Hz}$ the amplitude is chosen as $0.3 \mathrm{~A}$, while for the range 1000 to $3000 \mathrm{~Hz}$ it is $0.8 \mathrm{~A}$.

4. A time windows of 0.3 seconds is recorded, with a sampling rate of $100 \mu \mathrm{s}$, after the output has reached steady state.

5. Each amplifier unit is excited independently one by one.

\subsubsection{Complete plant data set}

In this data set the signals $\mathrm{u}$ and $\mathrm{y}$, the input-output data of the plant, is present. The signals are defined in Sec. 2.2. As explained in Sec. 2.2, the plant is $2 \times 2$ MIMO system in a single plane ( $x$ or $y$ ). The methodology for conducting stepped sine experiment for such a system is explained in Sec. 4.4.1. It consists of two separate excitation process, one for each input. Then the same procedure is repeated for the other plane, which is another $2 \times 2 \mathrm{MIMO}$ system. A single excitation process is designed as follows.

1. The frequency range is up to the second eigenfrequency of the rotor (10 to $1000 \mathrm{~Hz})$.

2. The frequency grid is logarithmically spaced over 200 points.

3. The amplitude profile is varied appropriately such that the rotor displacement is kept within the linear region, and also to compensate for the amplifier roll-off. (The exact amplitudes at each frequency point were unrecoverable from the data set.)

4. A time window of approximately 1 second is recorded, with a sampling rate of $100 \mu s$, after the output has reached steady state.

One important check to perform on the complete plant data set is to see if the rotor displacement is within the linear range. This can be confirmed by plotting the amplitudes of the output signal (rotor displacement) at all excitation frequencies. Figure 5.4 shows such a plot and it can be seen that the rotor displacement is within the linear range of $80 \mu \mathrm{m}$. 


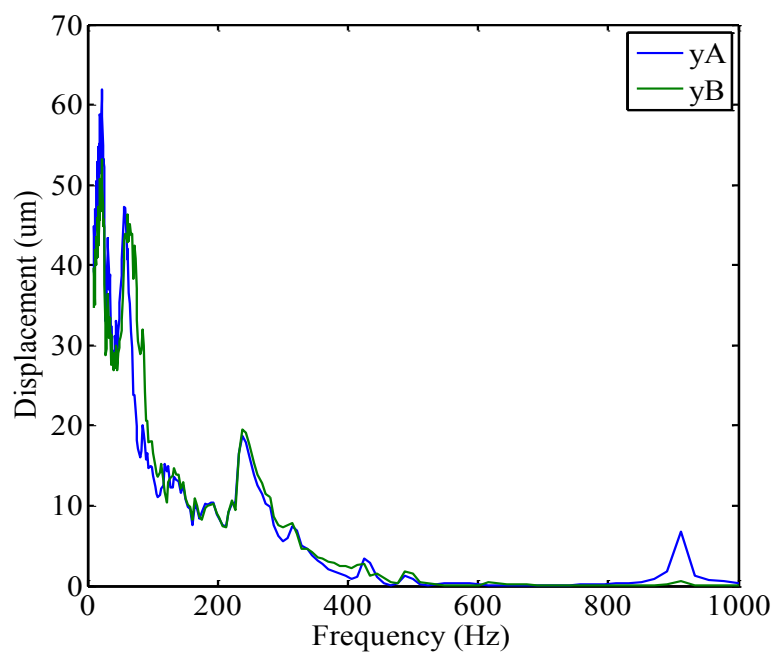

Figure 5.4 Rotor displacement at sides A and B

\subsection{Results}

In this section the results of the identification procedure which was applied to the experimental data is presented in the order of the procedural steps. Although PEM (ssest ()) has been chosen for identification, subspace method (n 4 sid () ) also has been tried and compared with the former. The identified state-space models are listed in Appendix A.

The PEM method such as ssest () involves an optimization algorithm. It requires search algorithms for searching in the parameter vector space. MATLAB provides the following choices for search algorithms: Gauss-Newton (gn), Adaptive Gauss-Newton (gna), Levenberg-Marquardt ( $1 \mathrm{~m})$, least square non-linear from the Optimization toolbox (lsqnonlin) and gradient descent (grad). It has been found that search algorithm 'gn' is usually sufficient to converge to the minimum. 'gna' and ' $1 \mathrm{~m}$ ' converged to the same values. 'Isqnonlin' and 'grad' sometimes required significantly more number of iterations to converge. For these reasons 'gn' was chosen as the search algorithm. The iterations continued until no further improvements were detected. All other settings for ssest () were left at default values. The different options can be found in [31].

\subsubsection{Step 1: Identification experiment}

As mentioned earlier, an experiment was not conducted but rather existing data was used for the procedure. The details of the data were presented in Sec. 5.2. The data set was the timedomain record of a stepped sine experiment which is very much what would have resulted had a new experiment been conducted.

\subsubsection{Step 2: Generation of the nonparametric model}

The goal of Step 2 was to generate frequency response data, resulting in a nonparametric model. The stepped sine experiment produced two sets of time domain data, one for the amplifier block and the other for the complete system. As discussed in Sec. 4.2.2, ETFE method was chosen to convert the time domain data into frequency domain data. 
Fig. 5.5 shows the nonparametric model of the two amplifiers, pertaining to the $x$-plane. The two amplifiers at sides A and B were treated as independent SISO systems. The units of signals $\mathrm{u}$ and $\mathrm{i}$ is $\mathrm{A}$ (ampere). The frequency range of 0 to $3 \mathrm{Khz}$ is sufficient since it captures the amplifier attenuation of up to $-15 \mathrm{~dB}$.
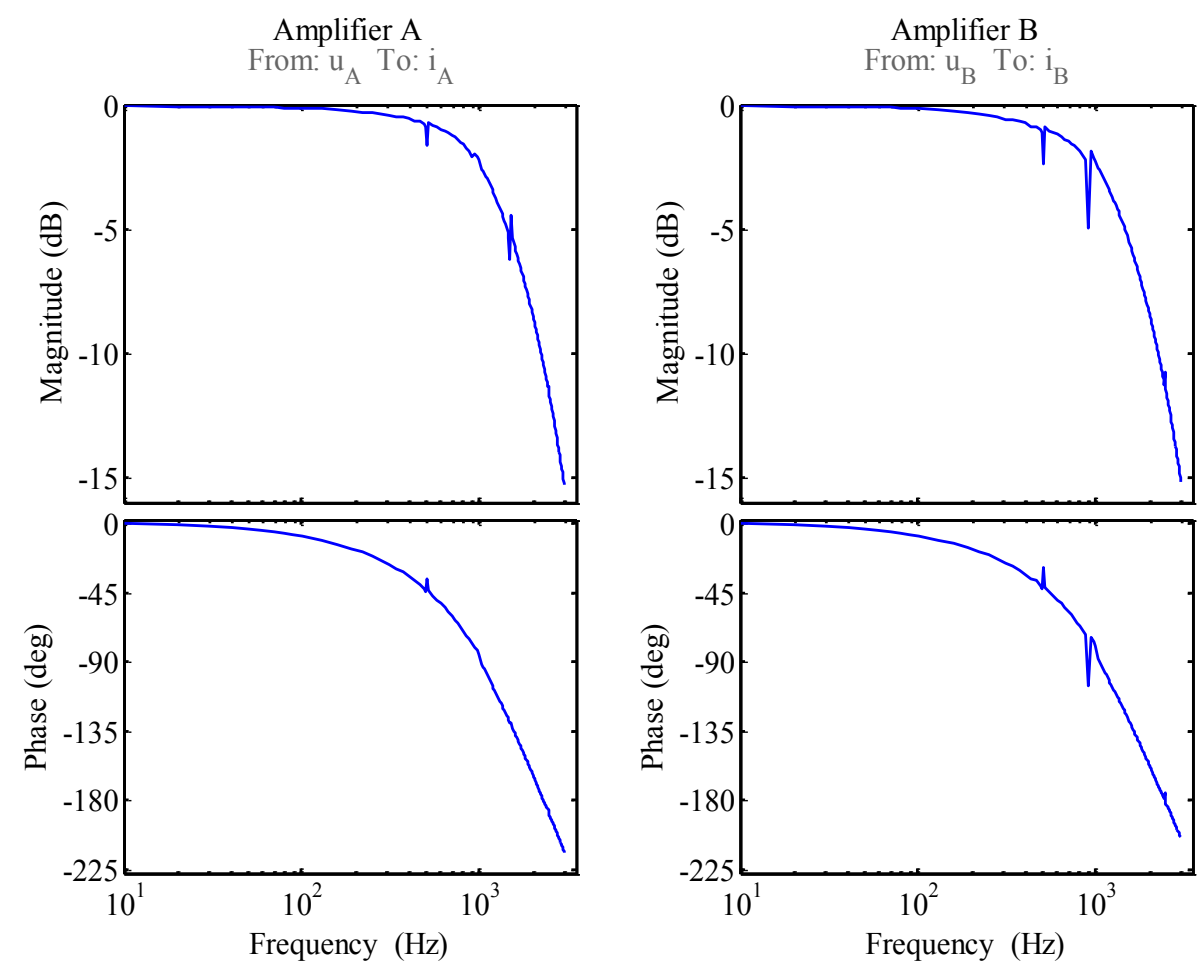

Figure 5.5 Nonparametric model of amplifiers in $x$-plane. $\mathbf{u}$ and $\mathbf{i}$ represent control and coil current signals, respectively, of the amplifier. Subscripts A and B represent the two AMBs.

The nonparametric model of the complete plant is shown in Fig. 5.6. The frequency response data covers a range which includes two resonance peaks. These peaks correspond to the two flexible modes. The first resonance peak appears to be split for some reason. The units of $u$ and $y$ are A (ampere) and $m$ (meter) respectively.

\subsubsection{Step 3: Identification of the amplifier}

The amplifier was identified using ssest ( ) function in IDENT. This function implements PEM method preceded by a preliminary subspace stage. For each amplifier, A and B, a first order state-space model in modal canonical form, was chosen. The nonparametric model for the amplifiers, presented in the previous subsection, was used as the data set. Fig. 5.7 shows the identified model with respect to the nonparametric model. The fit of the identified model is listed in Table 5-2.

It can be observed that the fit of the model is not good for either of the amplifiers. With such poor fitness, a re-identification with an updated model, this time a second order system, was attempted. Fig. 5.8 shows the results. A much better fit can be observed for a second order model. Table 5-2 lists the fit-defined as normalized root mean square error [31]-of the new model. Further increase in the model order did not yield any improvement. 


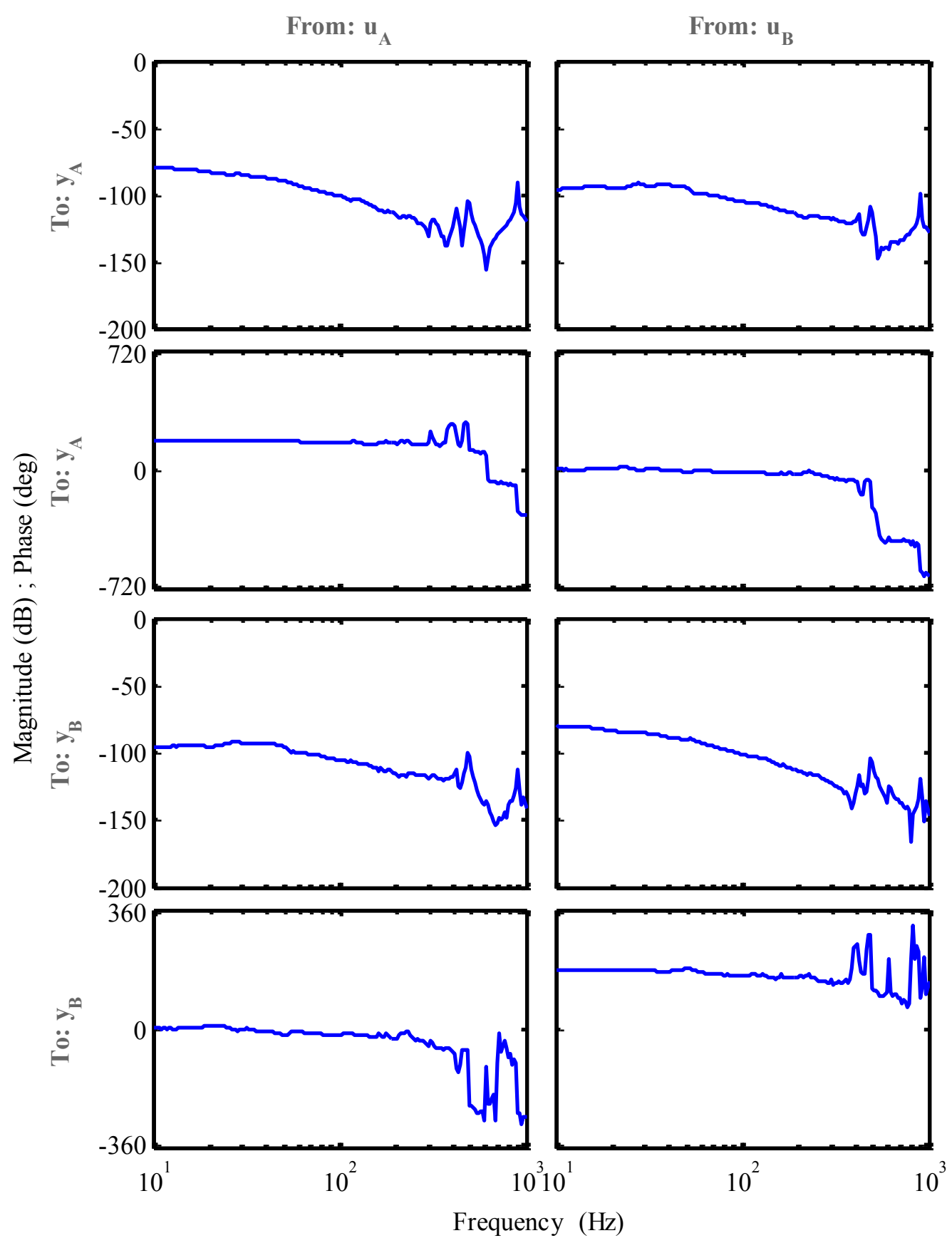

Figure 5.6 Nonparametric model of full plant ( $x$-plane). $\boldsymbol{u}$ and $\boldsymbol{y}$ represent control current and rotor displacement signals, respectively. Subscripts A and B represent the two AMBs.

Table 5-2 Fit of amplifier models

\begin{tabular}{ccc}
\hline Amplifier & First order & Second order \\
\hline A & $32 \%$ & $60 \%$ \\
B & $33 \%$ & $59 \%$ \\
\hline
\end{tabular}



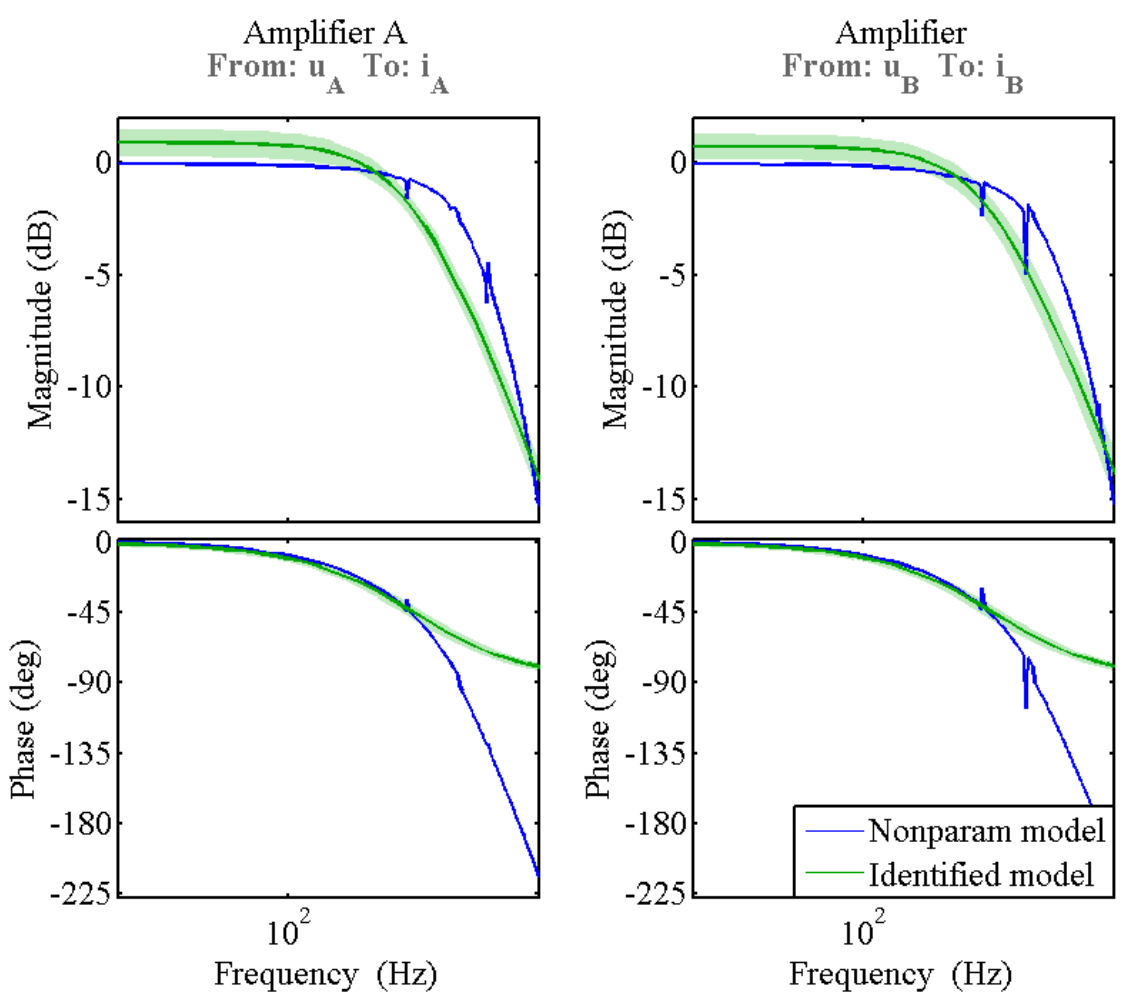

Figure 5.7 Identified amplifier model ( $x$-plane) with $99 \%$ confidence region. Amplifier A and B identified as independent first order SISO.
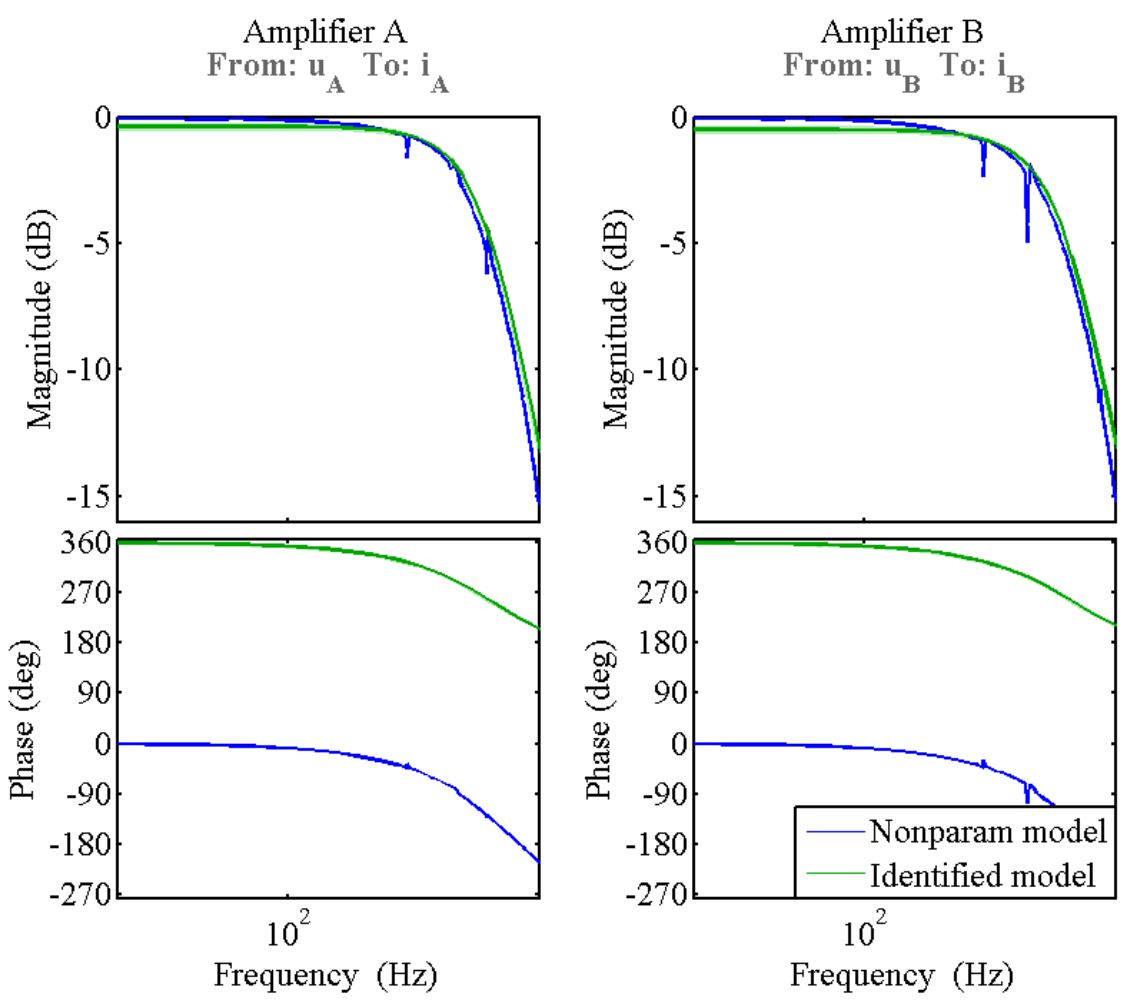

Figure 5.8 Identified amplifier model ( $x$-plane) with 99\% confidence region. Amplifier A and B identified as independent second order SISO. 


\subsubsection{Step 4: Identification of the rigid-rotor model and the AMB stiffness constants}

Grey-box identification of the rigid body model was done in this step. The nonparametric model was adjusted to exclude all flexible modes. After removing the resonance peaks the effective frequency range was from $10 \mathrm{~Hz}$ to $250 \mathrm{~Hz}$. Then, the model structure in (2.12) was setup in MATLAB and known/nominal values of various parameters (see Table 5-1) were used as the initial parameter vector. Gauss-Newton algorithm was used as the search algorithm. The fit of the identified model was $\left[\begin{array}{ll}91 & 61 \\ 62 & 90\end{array}\right] \%$. The frequency response of the identified model is shown in Fig. 5.9. It can be seen that the PEM method identified a better model compared to the analytical model.

The grey-box identification process was configured to output estimated values of the stiffness constants. The values of these constants can be seen in Table 5-3. IDENT also provided the standard deviations of the parameter set. It was discovered that the nominal values of the constants contained large uncertainties. This was probably due to the linearization errors in the system. According to [7] the uncertainty in the current stiffness constants can be as high as $50 \%$, while for the displacement stiffness it could be as high as $25 \%$ [1]. This, coincidently, matches with the current results.

Table 5-3 Identified stiffness constants ( $x$-plane)

\begin{tabular}{cccccc}
\hline Parameter & Units & Nominal value & Identified value & Std. Dev. & Error (\%) \\
\hline$K_{S A}$ & $N / m$ & $2.3 \times 10^{6}$ & $1.68 \times 10^{6}$ & $4 \times 10^{4}$ & 27 \\
$K_{S B}$ & $N / m$ & $2.3 \times 10^{6}$ & $1.73 \times 10^{6}$ & $4 \times 10^{4}$ & 25 \\
$K_{i A}$ & $N / A$ & 340 & 181 & 3.0 & 47 \\
$K_{i B}$ & $N / A$ & 340 & 186 & 3.2 & 45 \\
\hline
\end{tabular}

\subsubsection{Step 5: Identification of the flexible modes and the rotor parameters}

In this step the flexible modes of the system were identified using ssest () function. Since the rotor model had two flexible modes, the frequency response data consisted of only two resonant peaks in the nonparametric model. The effective frequnecy range was from $350 \mathrm{~Hz}$ to $1000 \mathrm{~Hz}$. A fourth order state-space model structure, in model form, was selected. The frequency response of the identified model is shown in Fig. 5.10. The flexible modes were successfully identified. The fit of the model was $\left[\begin{array}{ll}69 & 63 \\ 70 & 61\end{array}\right] \%$.

The rotor parameters were extracted from the identified model as discussed in Sec. 4.4.5. The values of these parameters are listed in Table 5-4. As expected the rotor structure was lightly damped, making the undamped and damped natural frequencies almost equal. The identified natrual frequencies matched well with the resonance peaks in the nonparametric model. 


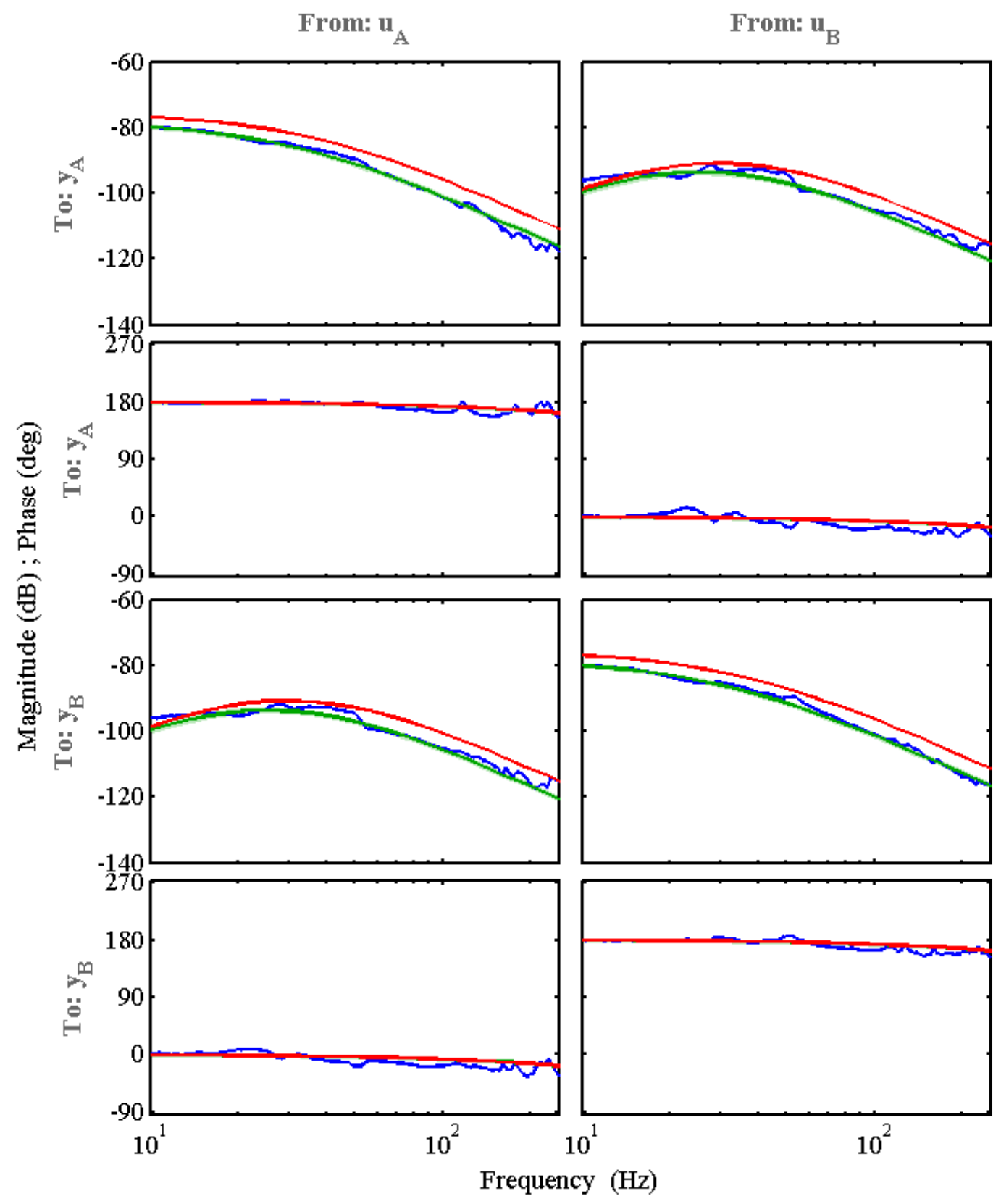

Figure 5.9 Frequency response of rigid model ( $x$-plane) with 99\% confidence region.

Table 5-4 Identified rotor parameters ( $x$-plane)

\begin{tabular}{cccc}
\hline Parameter & Units & Identified value & Std. Dev. \\
\hline$\omega_{n 1}$ & $\mathrm{rad} / \mathrm{s}$ & 3097 & - \\
$\zeta_{1}$ & - & 0.001 & - \\
$\omega_{n 2}$ & $\mathrm{rad} / \mathrm{s}$ & 5708 & - \\
$\zeta_{2}$ & - & 0.001 & - \\
\hline
\end{tabular}




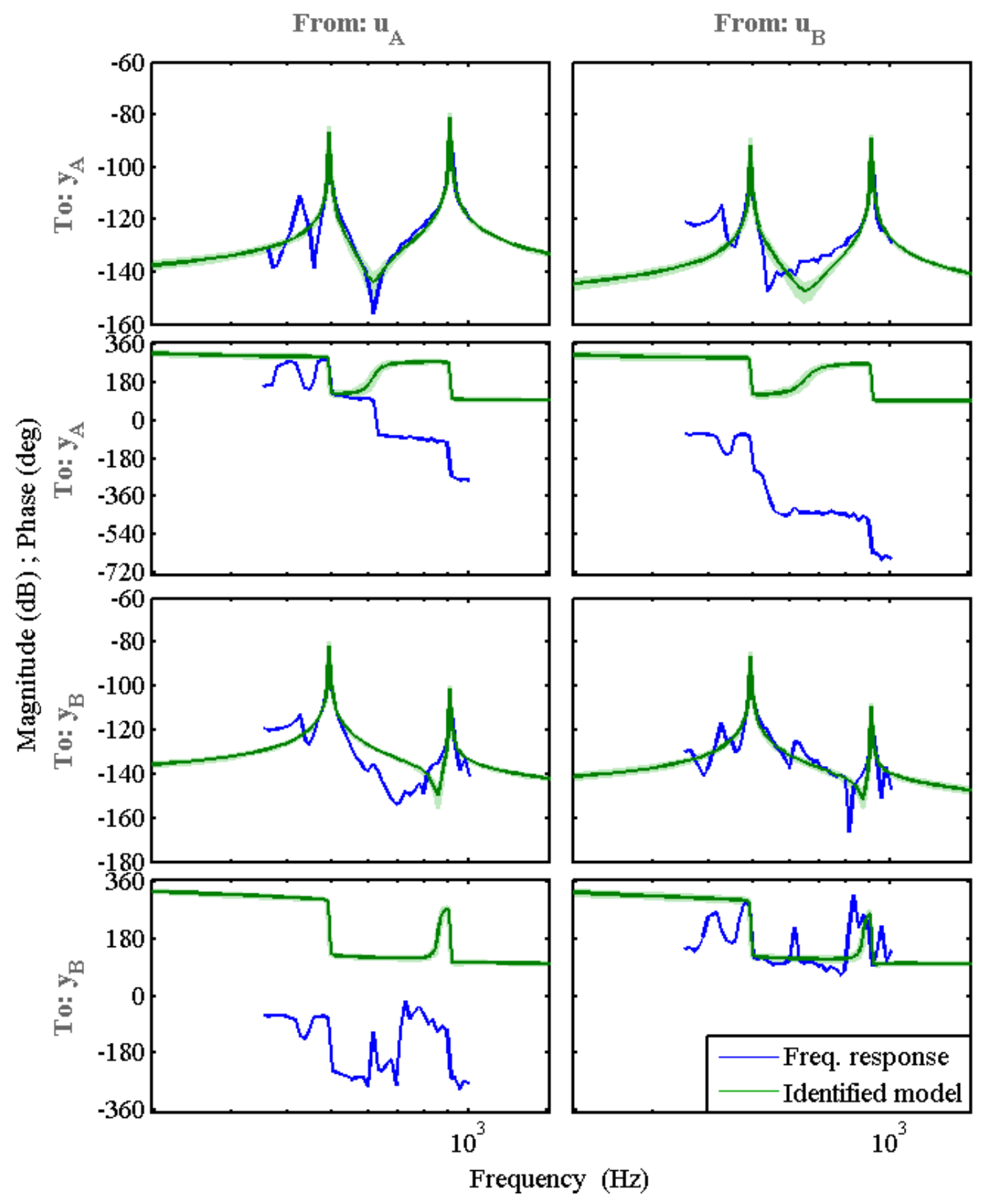

Figure 5.10 Frequency response of flexible modes model ( $x$-plane) with 99\% confidence region.

\subsubsection{Step 6: Combination of the rigid and flexible models}

The complete system model was obtained by combining the two models identified in Steps 4 and 5. The rigid model obtained in Step 4 was converted to modal from before the combination. The flexible mode model obtained in Step 5 was already in modal form. Figure 5.11 shows the identified model in comparison to the nonparametric model. The fit of the complete system model with the nonparametric model was $\left[\begin{array}{ll}92 & 70 \\ 71 & 91\end{array}\right] \%$. 


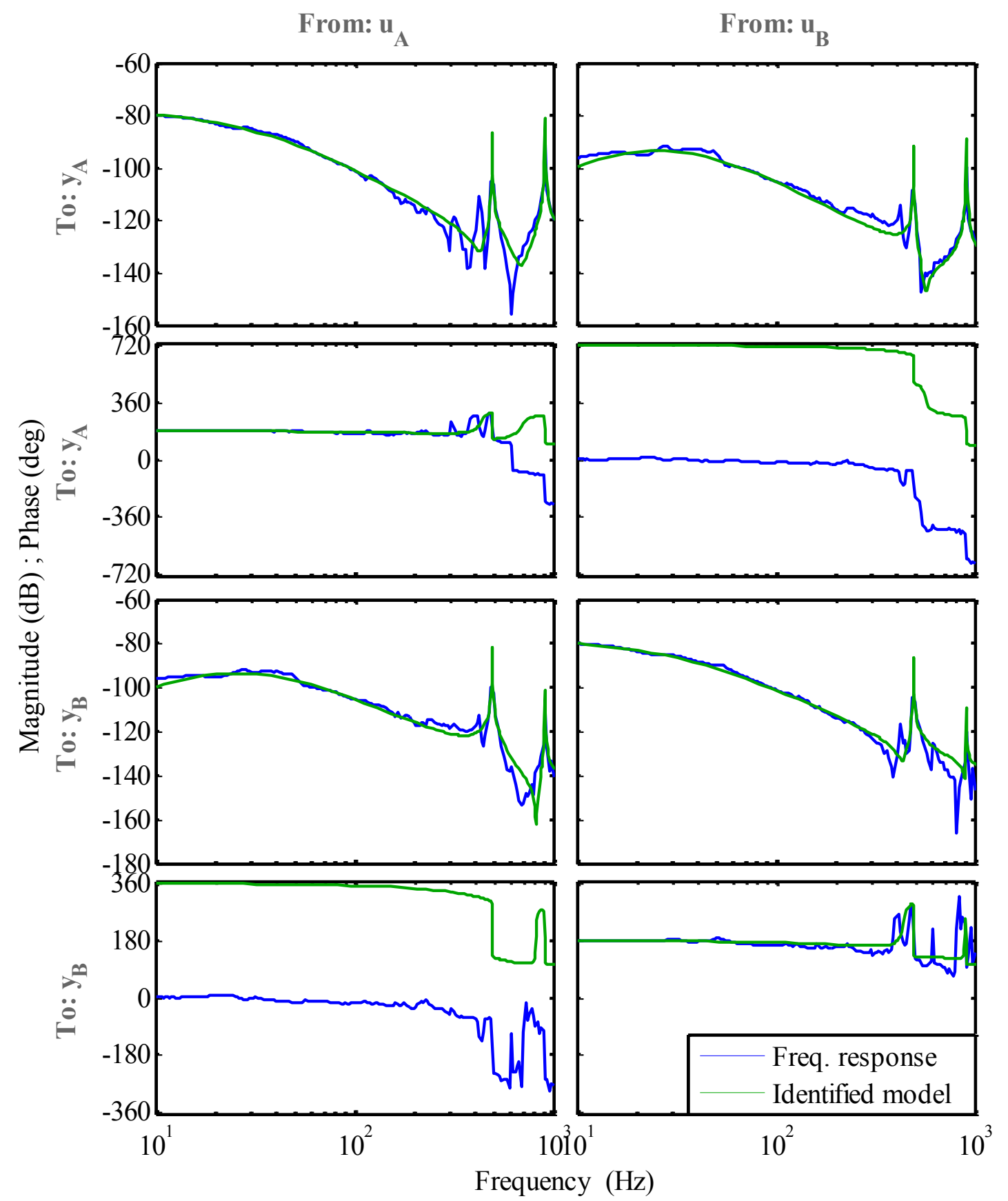

Figure 5.11 Frequency response of complete system model ( $x$-plane)

\subsubsection{Identification of the $y$-plane}

The $y$-plane of the system was identified by following exactly the same procedure. The identified model is listed in Appendix A. The amplifier model was assumed to be the same as the $x$-plane. The fit of the final model was $\left[\begin{array}{ll}94 & 74 \\ 67 & 92\end{array}\right] \%$. The identified parameters are presented in Table 5-5. The rotor parameters were expected to match with that of the $x$-plane and it did with very little variation $(\sim 0.002 \%)$. The stiffness constants were slightly different. This may be due to variations in mechanical systems or the electromagnets. 
Table 5-5 Identified parameters constants for $y$-plane

\begin{tabular}{cccccc}
\hline Parameter & Units & Nominal value & Identified value & Std. Dev. & Error (\%) \\
\hline$K_{S A}$ & $N / m$ & $2.3 \times 10^{6}$ & $1.69 \times 10^{6}$ & $3 \times 10^{4}$ & 26 \\
$K_{S B}$ & $N / m$ & $2.3 \times 10^{6}$ & $1.88 \times 10^{6}$ & $4 \times 10^{4}$ & 18 \\
$K_{i A}$ & $N / A$ & 340 & 184 & 2.3 & 46 \\
$K_{i B}$ & $N / A$ & 340 & 198 & 3.3 & 42 \\
$\omega_{n 1}$ & $\mathrm{rad} / \mathrm{s}$ & - & 3091 & - & - \\
$\zeta_{1}$ & - & - & 0.001 & - & - \\
$\omega_{n 2}$ & $\mathrm{rad} / \mathrm{s}$ & - & 5701 & - & - \\
$\zeta_{2}$ & - & - & 0.001 & - & - \\
\hline
\end{tabular}

\subsubsection{Comparison of PEM and subspace methods}

The procedure was designed with PEM as the identification method. But subspace method was also tried for the sake of comparison. Table 5-6 summarizes the comparison between PEM and subspace methods when used to identify the various sub models. The comparison is in terms of fit to the frequency response data or the nonparametric model. It can be seen that with the exception of the amplifier modes PEM does a better job. Unstructured black-box identification of the complete plant model produces more or less the same fit, with PEM having a slight lead. It can also be noticed that the unstructured model is slightly better than the model identified by the procedure, but with the risk of identification of spurious modes and lack of proper structure.

Table 5-6 Fitness comparison between PEM and subspace methods

\begin{tabular}{|c|c|c|c|c|}
\hline Model & \multicolumn{2}{|c|}{$\begin{array}{c}\text { PEM } \\
(\text { ssest }())\end{array}$} & \multicolumn{2}{|c|}{$\begin{array}{c}\text { Subspace } \\
(\mathrm{n} 4 \mathrm{sid}()) \\
\end{array}$} \\
\hline Amplifier & {$\left[\begin{array}{c}60 \\
0\end{array}\right.$} & $\left.\begin{array}{c}0 \\
59\end{array}\right] \%$ & {$\left[\begin{array}{c}60 \\
0\end{array}\right.$} & $\left.\begin{array}{c}0 \\
59\end{array}\right] \%$ \\
\hline $\begin{array}{l}\text { Rigid modes } \\
\text { (Grey-box) }\end{array}$ & {$\left[\begin{array}{l}91 \\
62\end{array}\right.$} & $\left.\begin{array}{l}61 \\
90\end{array}\right] \%$ & {$\left[\begin{array}{l}90 \\
41\end{array}\right]$} & $\left.\begin{array}{l}53 \\
86\end{array}\right] \%$ \\
\hline Flexible modes & {$\left[\begin{array}{l}69 \\
70\end{array}\right.$} & $\left.\begin{array}{l}63 \\
61\end{array}\right] \%$ & {$\left[\begin{array}{l}51 \\
57\end{array}\right]$} & $\left.\begin{array}{l}48 \\
49\end{array}\right] \%$ \\
\hline $\begin{array}{l}\text { Complete plant } \\
\text { (unstructured) }\end{array}$ & {$\left[\begin{array}{l}93 \\
74\end{array}\right.$} & $\left.\begin{array}{l}76 \\
91\end{array}\right] \%$ & {$\left[\begin{array}{l}93 \\
72\end{array}\right.$} & $\left.\begin{array}{l}75 \\
91\end{array}\right] \%$ \\
\hline $\begin{array}{c}\text { Complete plant } \\
\text { (procedure) }\end{array}$ & {$\left[\begin{array}{l}92 \\
71\end{array}\right.$} & $\left.\begin{array}{l}70 \\
91\end{array}\right] \%$ & & - \\
\hline
\end{tabular}

\subsection{Model validation}

In this section the results of identification are validated by applying some basic validation methods.

\subsubsection{Residual analysis}


Residual analysis in IDENT has two tests, the whiteness test and the independence test. Accordingly, IDENT generates estimated power spectrum of the residuals for each output and transfer-function amplitude from input to the residuals, for each input-output pair, respectively. According to [31], for frequency domain output-error models, whiteness test of the residual power spectrum is not so important, instead the independence test is more meaningful. Furthermore, both the tests are confirmed if the estimated value lies within a specific confidence interval. A confidence interval of $99 \%$ was selected. Fig. 5.12 shows the residual plots for the different identification tests.

It can be seen from Fig. 5.12 that not only the residuals at outputs are almost white, but in general the plots are within $99 \%$ confidence interval that was set in IDENT. Here confidence interval corresponds to the range of residual values with a specific probability of being statistically insignificant for the system [31]. Residual analysis indicates good quality of identification.
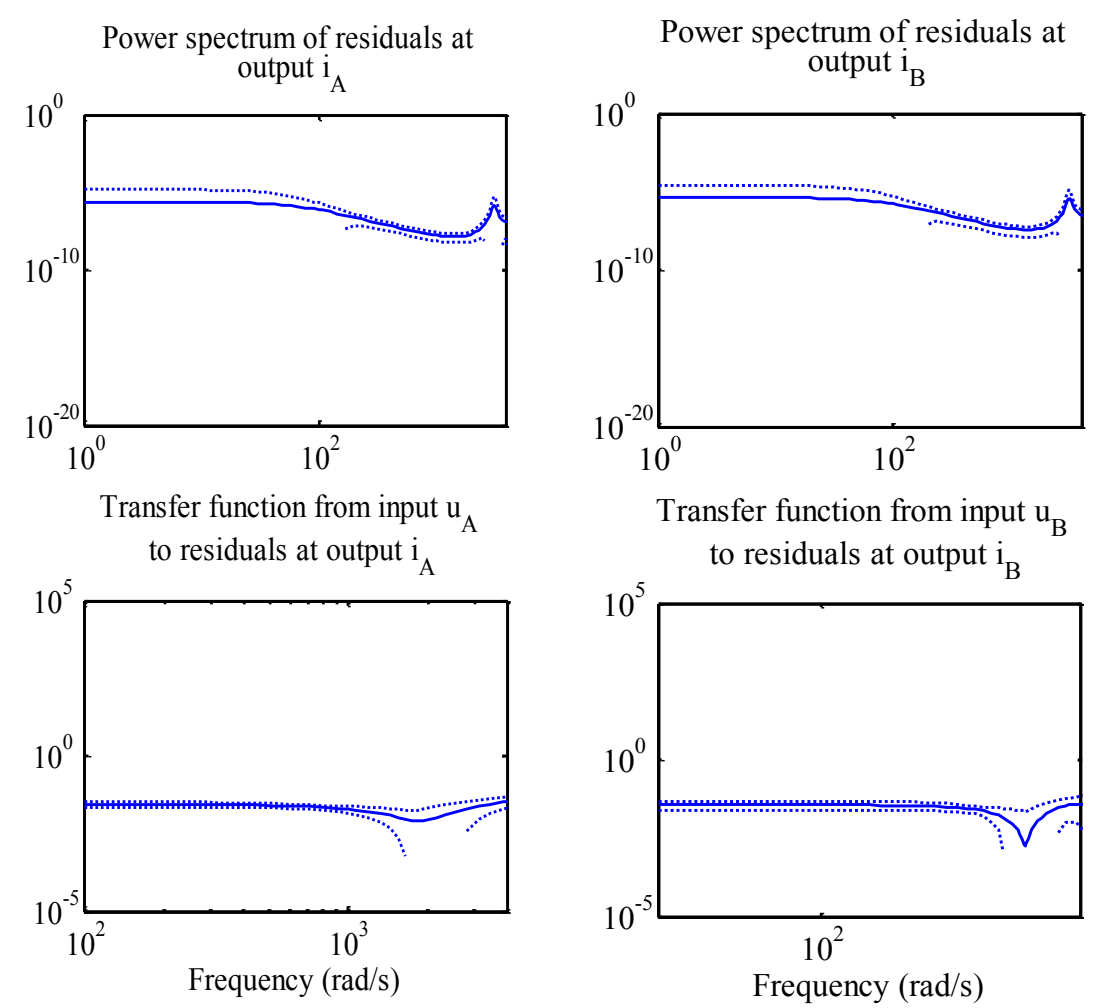

Figure 5.12a Residual analysis of amplifier identification (logarithmic scale). The dotted line represents $99 \%$ confidence interval. 

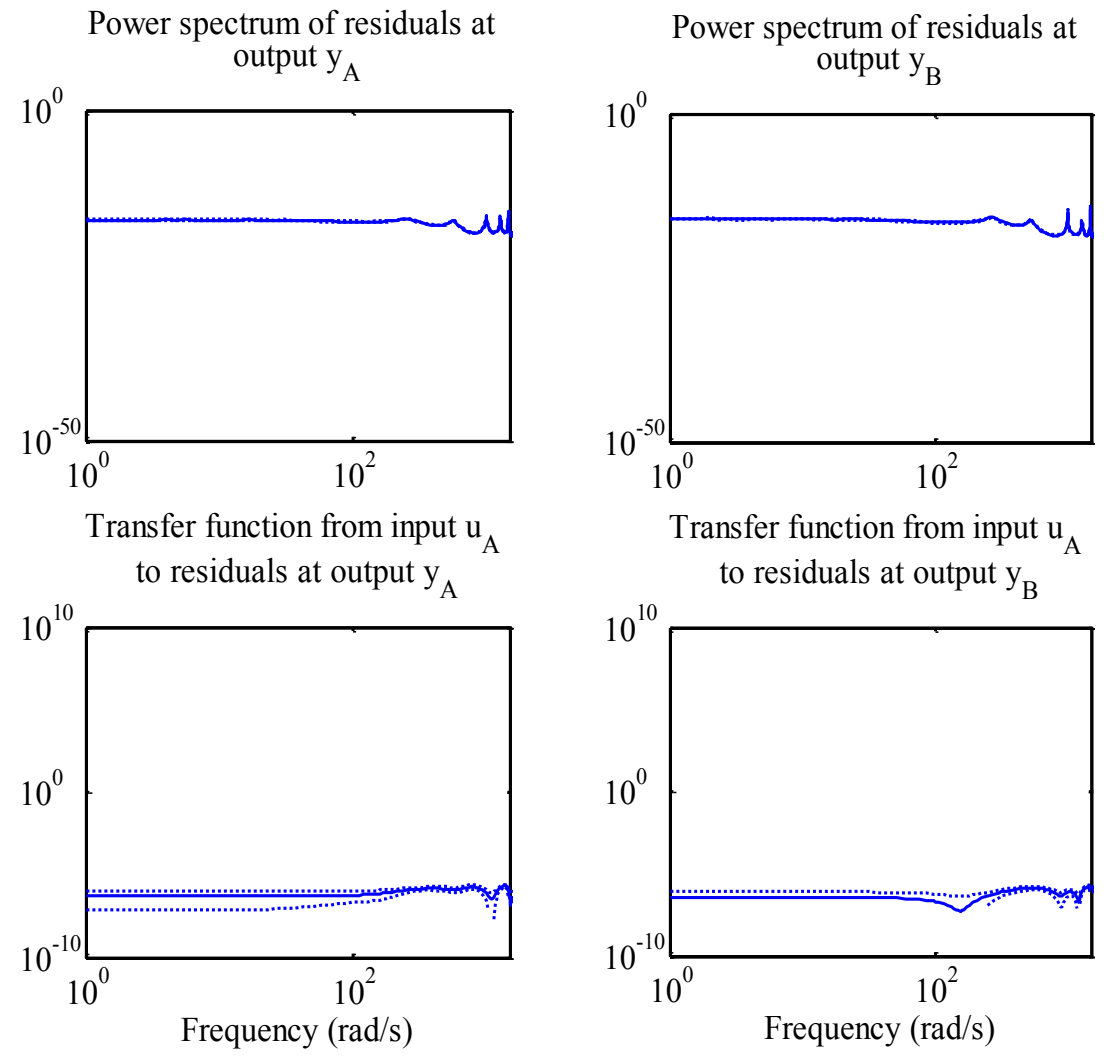

Figure 5.12b Residual analysis of rigid model identification (logarithmic scale). The dotted line represents $99 \%$ confidence interval.
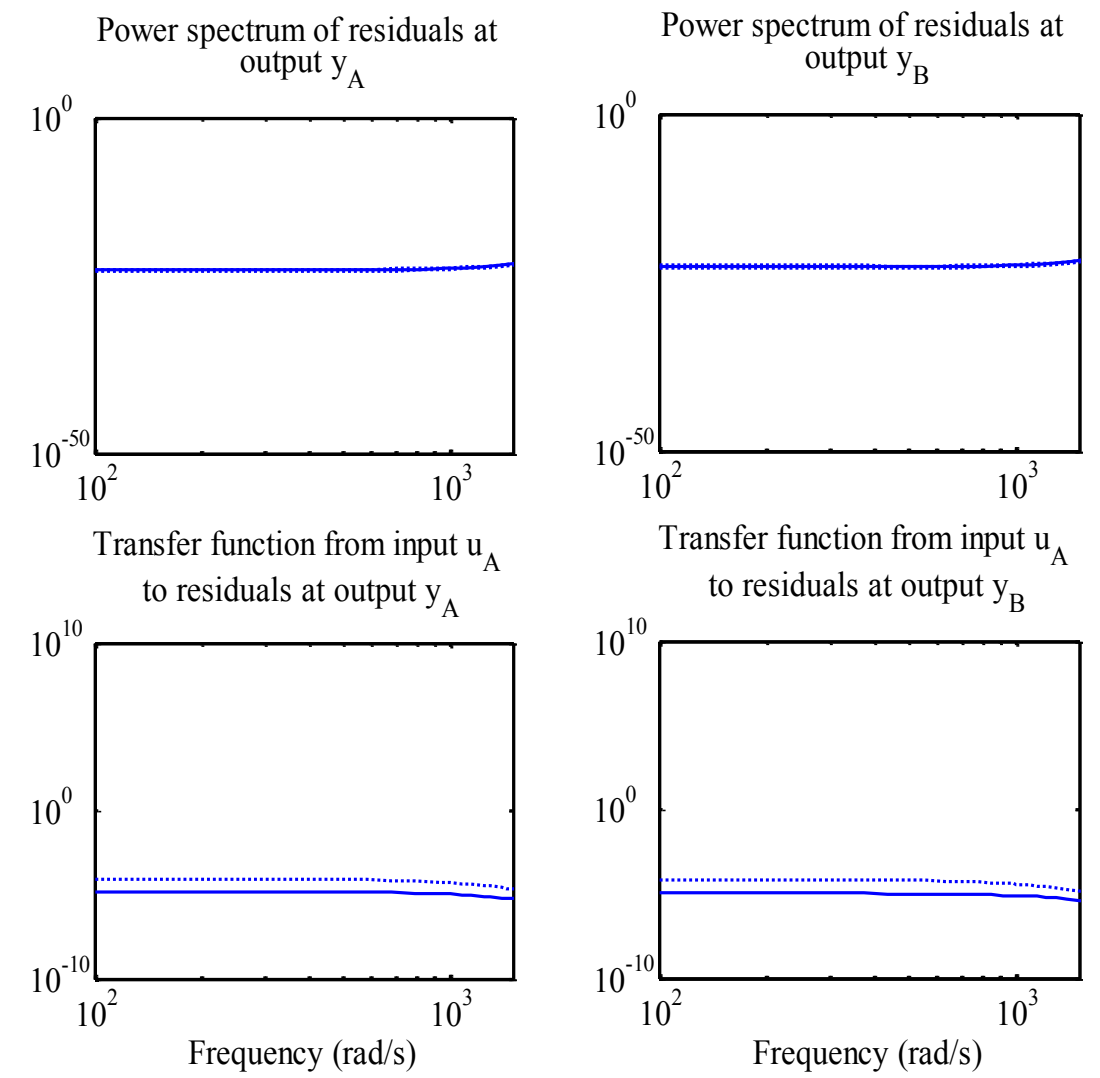

Figure 5.12c Residual analysis of flexible mode identification (logarithmic scale). The dotted line represents $99 \%$ confidence interval. 


\subsubsection{Uncertainty of the identified models}

IDENT computes and stores covariance matrix of the parameter vector after an identification. The covariance matrix is used to compute the uncertainty for the frequency response plots that is generated by the bode ( ) command. Assuming Gaussian distribution for the random variables, the uncertainty is specified as a confidence region with a specific probability measure. This allows the user to visually view the uncertainty for a specific confidence interval setting. This is one way to assess the quality of identification.

Even with a $99 \%$ setting, the confidence regions of most of the frequency response plots (Fig. 5.7 - 5.10) were hard to distinguish from the estimate itself. With sufficient zooming in, the regions were visible. This indicated good quality of estimation.

\subsubsection{Frequency response}

Ideally, this should have been done with some kind of cross-validation, wherein the frequency responses (bode plots) of the identified model and the (independent) true system response are compared. Due to the inaccessibility of the test system, an independent validation data set was not available for this study. However, a look at Fig. 5.7 to Fig. 5.10 reveals that the identified models fit rather well to the measured frequency response data (or the nonparametric model). It can be seen that the model does capture the prominent features of the data, for example the resonance peaks. The fit of the complete plant model to the frequency response data obtained in Step 6 (Sec. 5.3.6) was quite good.

\subsection{Summary}

In this chapter the experimental system and the experimental results were described. The results presented in detail are only for the $x$-axis. Both $x$ and $y$ axis models that were identified are listed in Appendix A. PEM method, available as the function ssest ( ) in IDENT, was used for identification.

The identified models were validated primarily by residual analysis. The quality of identified models was found to be reasonably good. Separate identification of amplifiers led to model updating from first order to second order. The parameters identified were found to differ significantly from their nominal values. A comparison of PEM with subspace methods revealed that the former was better. 


\section{Chapter 6}

\section{Discussion and Conclusion}

This chapter contains key discussions relevant to the work and the results presented in previous chapters. It further provides some conclusion and suggestions for future work.

\subsection{Discussion}

\subsubsection{Separate identification of the system modes}

The strategy of identifying the underlying modes of the system rather that the whole system as a black-box is key to this work. This had multiple benefits, namely, it enabled structured identification, possibly improved the fit of the complete model, and finally allowed the identification of parameters without requiring FEM. A structured identification allows for the direct comparison of the identified model with its analytical model. Also, it has the potential of making the identification procedure somewhat modular, for example if the system needs to be operated only in subcritical mode, then the system model could be easily updated by removing the flexible modes.

\subsubsection{Cross-validation}

Lack of cross validation, in general, could lead to over-fitting [31]. This is because an increase in model order would usually increase the fit for a particular data set. However this is not a big danger for this work, since the model structure is chosen based on a priori knowledge of the system (analytical model) and not random guessing. The fact that the rotor parameters (resonance frequencies and damping ratios) were almost identical for both the planes is a good indication that the true system has been identified. This is because, unlike the stiffness constants, the rotor parameters should be the same in both the planes.

\subsubsection{Fitness of the model}

So far the fit of the identified model to the data has been expressed in percentage. IDENT defines the fit of the model to the data as normalized root mean square error (NRMSE):

$$
\operatorname{NRMSE}=100\left(1-\frac{|| y-\hat{y} \|}{|| y-\operatorname{mean}(y) \mid}\right)
$$

where $y$ is the validation data and $\hat{y}$ is the model output. For frequency-domain identification, NRMSE is calculated by comparing the complex frequency response. In addition to NRMSE, 
IDENT also provides Akaike's Final Prediction Error (FPE) and Mean Square Error (MSE) for relative comparison of models [31].

Since the system is MIMO $(2 \times 2)$ the fit is a $2 \times 2$ matrix with each element representing the corresponding transfer function. The rows and columns of the fitness matrix represent the outputs and inputs of the system, respectively.

$$
F i t=\left[\begin{array}{ll}
f_{A A} & f_{A B} \\
f_{B A} & f_{B B}
\end{array}\right]
$$

For example, bottom-left element represents the transfer function between output B and input A. It can be seen from the results in Chapter 5 that $f_{A A}\left(\right.$ or $\left.f_{B B}\right)$ is higher than $f_{A B}$ (or $\left.f_{B A}\right)$. This is due to lower SNR of the latter. Intuitively this means that an input at side A primarily causes motion at side $\mathrm{A}$ itself. It also causes motion at side $\mathrm{B}$, but to a much lesser extent.

\subsubsection{Model updating}

Amplifier modes turned out to be second order systems, and therefore required model updating. The reason for this is unknown and was not investigated. It is probably due to something other than a pure proportional control for the current controller, which could not be verified. However, this will not be a problem for a known system. For the rest of the system, the model structure selected from analytical model is sufficient and there is little risk of under modeling. The number of state variables was increased by two in the updated models, but otherwise had no impact on the procedure.

\subsubsection{Gyroscopic effect}

It is true that the thesis was limited to nonrotating rotor in order to simply the identification problem. A rotating rotor could introduce gyroscopic effects which would make the system linear time variant (LTV). However, as long as the gyroscopic effects are negligible, for example in the case of lighter loads, the procedure would still work. A comprehensive method for identification of gyroscopic matrix of the system can be found in [6].

\subsubsection{Automated identification}

Automated identification is attractive for commissioning. The primary challenge with regard to automation is the implementation of the identification experiment. Design and execution of stepped sine experiment involves choosing the frequency range, frequency steps and the amplitude profile. Automation stepped sine could be based on the principles presented in Sec. 3.4. Other issues where human intervention was required were the choice of number of flexible modes and choice of the frequency regions for rigid/flexible modes. These issues limit the automation of the procedure. Even so, once a system is commissioned and all necessary information is acquired, the procedure can be automated for future diagnostic purposes.

\subsubsection{Noise model}


A simpler model structure (output-error) was chosen because of the limitation of IDENT. IDENT supports only output-error models for frequency domain identification. Residual analysis indicates good whiteness characteristics, and therefore it appears that output error model was sufficient.

\subsubsection{Identifiability of an extended parameter set}

The set of identifiable parameter set for the grey-box identification in Step 4 was limited to the stiffness constants and the rotor parameters. This was what the requirement of identification for commissioning was to begin with. However, the grey-box identification step is possible only if all the necessary system parameters in Table 5-1 are available at the time of commissioning. Two parameters are difficult to obtain, namely, the moment of inertia and center of gravity of the rotor. It would be great to include these parameters into the identifiable set and then let the grey-box step identify them. But then the new extended set of parameters has to be proven to be identifiable. An attempt to do so using similarity transformation approach was unsuccessful as the algebraic equations became too complex. Another method to prove structural identifiability is required, but was not pursued. Preliminary trials assuming the extended set to be identifiable showed good results, indicating that the extra parameters are at least locally identifiable.

\subsubsection{Controller synthesis}

As mentioned in Sec. 1.4, a decentralized PID controller, one based on the analytical model, is currently deployed on the AMB system. A new controller could be synthesized with the identified model and it's performed measured. This was not done as a part of the thesis. But previous experience has revealed that a static gain equal to 2, when applied to the controller based on the analytical model, was able to stabilize the system which was otherwise oscillatory [32]. This correlates with the $45-50 \%$ error in the current stiffness constant, and provides good indication that the identified model would lead to a more accurate controller.

\subsection{Conclusion}

A procedure for identifying a linear state-space model of a flexible-rotor AMB (nonrotating), suitable for commissioning, has been developed. The planes $x$ and $y$ were identified independently. A frequency-domain approach was preferred over time-domain approaches, for noise reduction advantages. Structured identification in the light of an analytically derived model structure was preferred over unstructured black-box identification. A stepped sine experiment was chosen for its high SNR. The procedure is intentionally made independent of FEM in order to be suitable for commissioning.

Various modes of the system viz., amplifier modes, rigid and flexible modes of the rotor were identified separately and then combined. The amplifier and flexible mode models were identified in modal canonical form using standard PEM method available in MATLAB without the need for an explicit model structure. On the other hand, the rigid model was identified using a grey-box identification function in MATLAB which required an explicit model structure 
(analytical model). The frequency-domain data set, obtained through nonparametric identification, was split into two regions to be used for separate identification of rigid and flexible modes.

Validation showed good quality of the identified models. PEM and subspace methods were tried and the former was proven to be better in terms of fit. Large errors, probably due to linearization, were discovered for the nominal values of AMB stiffness constants. This proves the importance of system identification for effective control of AMB systems.

\subsection{Future work suggestions}

\subsubsection{Cross validation}

It is highly recommended to carry out fresh experiment on the system and perform crossvalidation. Although the results in this work are satisfactory, system identification with only one data set is not safe at all.

\subsubsection{Identification of a gyroscopic model}

The procedure developed in this work has to be augmented with identifying gyroscopic models. Without such extension it would not be fully suitable for commissioning. This requirement can be relaxed only if it is known in advance that gyroscopic effects are negligible for a particular application.

\subsubsection{Identification of MOI and COG}

As discussed in Sec. 6.1.8, extension of the set of identifiable parameters to include moment of inertia (MOI) and center of gravity (COG) of the rotor would be extremely useful for commissioning.

\subsubsection{Automation of the procedure}

An automated stepped sine excitation based on the principles discussed in Sec. 3.4 could be developed and verified. 


\section{Appendix A}

\section{Identified Models}

\section{A.1 Identification of $x$-plane}

The models identified in the experiment are listed below. The models presented here are continuous-times models.

\section{A.1.1 Amplifier model}

$$
\begin{aligned}
\mathrm{A}_{\mathrm{a}} & =\left[\begin{array}{cccc}
-7458 & 5462 & 0 & 0 \\
-5462 & -7458 & 0 & 0 \\
0 & 0 & -8008 & 5364 \\
0 & 0 & -5364 & -8008
\end{array}\right] \\
\mathrm{B}_{\mathrm{a}} & =\left[\begin{array}{ccc}
160.8 & 0 \\
1.908 \mathrm{e}+04 & 0 \\
0 & 651.7 \\
0 & 1.851 \mathrm{e}+04
\end{array}\right] \\
\mathrm{C}_{\mathrm{a}} & =\left[\begin{array}{cccc}
0.9013 & -0.0933 & 1 & 1 \\
1 & 1 & 0.9943 & -0.1123
\end{array}\right] \\
\mathrm{D}_{\mathrm{a}} & =\left[\begin{array}{ll}
0 & 0 \\
0 & 0
\end{array}\right]
\end{aligned}
$$

\section{A.1.2 Rigid-rotor model}

$$
\begin{aligned}
& \mathrm{A}_{\mathrm{r}}=\left[\begin{array}{cccccccc}
-7458 & 5462 & 0 & 0 & 0 & 0 & 0 & 0 \\
-5462 & -7458 & 0 & 0 & 0 & 0 & 0 & 0 \\
0 & 0 & -8008 & 5364 & 0 & 0 & 0 & 0 \\
0 & 0 & -5364 & -8008 & 0 & 0 & 0 & 0 \\
0 & 0 & 0 & 0 & 0 & 0 & 1 & 0 \\
0 & 0 & 0 & 0 & 0 & 0 & 0 & 1 \\
3.206 & -0.3319 & -2.207 & 0.2493 & 3.311 \mathrm{e}+04 & -2.071 \mathrm{e}+04 & 0 & 0 \\
-1.953 & 0.2022 & 3.429 & -0.3873 & -2.016 \mathrm{e}+04 & 3.218 \mathrm{e}+04 & 0 & 0
\end{array}\right] \\
& \mathrm{B}_{\mathrm{r}}=\left[\begin{array}{cc}
160.8 & 0 \\
1.908 \mathrm{e}+04 & 0 \\
0 & 651.7 \\
0 & 1.851 \mathrm{e}+04 \\
0 & 0 \\
0 & 0 \\
0 & 0 \\
0 & 0
\end{array}\right] \\
& \mathrm{C}_{\mathrm{r}}=\left[\begin{array}{cccccccc}
0 & 0 & 0 & 0 & 1.158 & 0 & 0 & 0 \\
0 & 0 & 0 & 0 & 0 & 1.163 & 0 & 0
\end{array}\right] \\
& \mathrm{D}_{\mathrm{r}}=\left[\begin{array}{ll}
0 & 0 \\
0 & 0
\end{array}\right]
\end{aligned}
$$




\section{A.1.3 Flexible mode model}

$$
\begin{aligned}
A_{f} & =\left[\begin{array}{cccc}
-6.071 & 5708 & 0 & 0 \\
-5708 & -6.071 & 0 & 0 \\
0 & 0 & -3.766 & 3097 \\
0 & 0 & -3097 & -3.766
\end{array}\right] \\
\mathrm{B}_{\mathrm{f}} & =\left[\begin{array}{ccc}
1.952 \mathrm{e}-05 & -9.098 \mathrm{e}-05 \\
0.001838 & 0.0007207 \\
-0.0008274 & -0.0004676 \\
-0.0001537 & -0.0001169
\end{array}\right] \\
\mathrm{C}_{\mathrm{f}} & =\left[\begin{array}{cccc}
0.07917 & -0.5903 & 0.3021 & 0.2827 \\
0.009627 & -0.0554 & 0.5053 & 0.528
\end{array}\right] \\
\mathrm{D}_{\mathrm{f}} & =\left[\begin{array}{ll}
0 & 0 \\
0 & 0
\end{array}\right]
\end{aligned}
$$

\section{A.1.4 Complete plant model}

$\mathrm{A}_{\mathrm{p}}=\left[\begin{array}{cccccccccccc}-7458 & 5462 & 0 & 0 & 0 & 0 & 0 & 0 & 0 & 0 & 0 & 0 \\ -5462 & -7458 & 0 & 0 & 0 & 0 & 0 & 0 & 0 & 0 & 0 & 0 \\ 0 & 0 & -230.4 & 0 & 0 & 0 & 0 & 0 & 0 & 0 & 0 & 0 \\ 0 & 0 & 0 & -110.5 & 0 & 0 & 0 & 0 & 0 & 0 & 0 & 0 \\ 0 & 0 & 0 & 0 & 230.4 & 0 & 0 & 0 & 0 & 0 & 0 & 0 \\ 0 & 0 & 0 & 0 & 0 & 110.5 & 0 & 0 & 0 & 0 & 0 & 0 \\ 0 & 0 & 0 & 0 & 0 & 0 & -8008 & 5364 & 0 & 0 & 0 & 0 \\ 0 & 0 & 0 & 0 & 0 & 0 & -5364 & -8008 & 0 & 0 & 0 & 0 \\ 0 & 0 & 0 & 0 & 0 & 0 & 0 & 0 & -6.071 & 5708 & 0 & 0 \\ 0 & 0 & 0 & 0 & 0 & 0 & 0 & 0 & -5708 & -6.071 & 0 & 0 \\ 0 & 0 & 0 & 0 & 0 & 0 & 0 & 0 & 0 & 0 & -3.766 & 3097 \\ 0 & 0 & 0 & 0 & 0 & 0 & 0 & 0 & 0 & 0 & -3097 & -3.766\end{array}\right]$

$\mathrm{B}_{\mathrm{p}}=\left[\begin{array}{cc}1.639 \mathrm{e}+04 & 0 \\ -9778 & 0 \\ 2.029 & -1.974 \\ 0.4452 & 0.4537 \\ 1.855 & -1.810 \\ 0.4265 & 0.4352 \\ 0 & -1.647 \mathrm{e}+04 \\ 0 & 8490 \\ 1.952 \mathrm{e}-05 & -9.098 \mathrm{e}-05 \\ 0.001838 & 0.0007207 \\ -0.0008274 & -0.0004676 \\ -0.0001537 & -0.0001169\end{array}\right]$

$\mathrm{C}_{\mathrm{p}}$

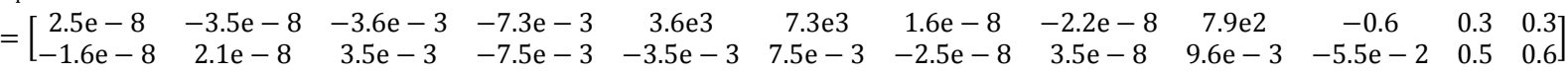

$\mathrm{D}_{\mathrm{p}}=\left[\begin{array}{ll}0 & 0 \\ 0 & 0\end{array}\right]$ 


\section{A.2 Identification of $y$-plane}

The complete plant model was

$\mathrm{A}_{\mathrm{p}}=\left[\begin{array}{cccccccccccc}-7458 & 5462 & 0 & 0 & 0 & 0 & 0 & 0 & 0 & 0 & 0 & 0 \\ -5462 & -7458 & 0 & 0 & 0 & 0 & 0 & 0 & 0 & 0 & 0 & 0 \\ 0 & 0 & -235.6 & 0 & 0 & 0 & 0 & 0 & 0 & 0 & 0 & 0 \\ 0 & 0 & 0 & -112.9 & 0 & 0 & 0 & 0 & 0 & 0 & 0 & 0 \\ 0 & 0 & 0 & 0 & 235.6 & 0 & 0 & 0 & 0 & 0 & 0 & 0 \\ 0 & 0 & 0 & 0 & 0 & 112.9 & 0 & 0 & 0 & 0 & 0 & 0 \\ 0 & 0 & 0 & 0 & 0 & 0 & -8008 & 5364 & 0 & 0 & 0 & 0 \\ 0 & 0 & 0 & 0 & 0 & 0 & -5364 & -8008 & 0 & 0 & 0 & 0 \\ 0 & 0 & 0 & 0 & 0 & 0 & 0 & 0 & -2.839 & 5701 & 0 & 0 \\ 0 & 0 & 0 & 0 & 0 & 0 & 0 & 0 & -5701 & -2.839 & 0 & 0 \\ 0 & 0 & 0 & 0 & 0 & 0 & 0 & 0 & 0 & 0 & -3.534 & 3091 \\ 0 & 0 & 0 & 0 & 0 & 0 & 0 & 0 & 0 & 0 & -3091 & -3.534\end{array}\right]$

$\mathrm{B}_{\mathrm{p}}=\left[\begin{array}{cc}1.639 \mathrm{e}+04 & 0 \\ -9778 & 0 \\ -2.034 & 2.134 \\ -0.4767 & -0.4603 \\ -1.856 & 1.953 \\ 0.4562 & 0.4411 \\ 0 & -8854 \\ 0 & 1.627 \mathrm{e}+04 \\ 0.0001265 & -6.069 \mathrm{e}-05 \\ 0.001998 & 0.0007642 \\ -0.000781 & -0.0004749 \\ -0.0001744 & -9.331 \mathrm{e}-05\end{array}\right]$

$\mathrm{C}_{\mathrm{p}}$

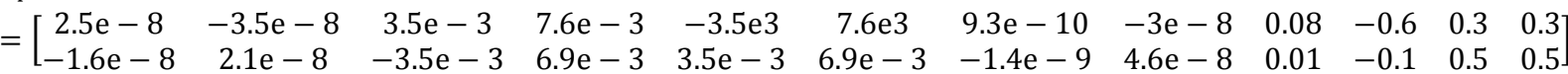

$\mathrm{D}_{\mathrm{p}}=\left[\begin{array}{ll}0 & 0 \\ 0 & 0\end{array}\right]$ 


\section{Appendix B}

\section{Structural Identifiability}

\section{B.1 Similarity transformation method}

The basic idea of similarity transformation method, for proving structural identifiablity, is that if there are no similarity transformations possible for the given parameterization of a statespace model, then the given model is unique and therefore identifiable. There will not be any danger of the identification method estimating parameters based on a similarity transformed model. The method is described in detail in [24].

Let the given parameterized model be

$$
\begin{aligned}
& \dot{x}=A(\theta) x+B(\theta) u \\
& y=C(\theta) x+D(\theta) u
\end{aligned}
$$

If the model is minimal, then there exists an invertible transformation matrix $T$ such that

$$
\begin{gathered}
A(\bar{\theta})=T A(\theta) T^{-1} \text { or } A(\bar{\theta}) T=T A(\theta) \\
B(\bar{\theta})=T B(\theta) \\
C(\bar{\theta})=C(\theta) T^{-1} \text { or } C(\bar{\theta}) T=C(\theta)
\end{gathered}
$$

where the new model, with $z=T x$,

$$
\begin{gathered}
T \dot{z}=A(\bar{\theta}) z+B(\bar{\theta}) u \\
y=C(\bar{\theta}) z+D(\bar{\theta}) u
\end{gathered}
$$

has the identical input-output behavior as the initial one.

If the only solution for (B.2) - (B.4) is $T=I$, the identity matrix, then the system is structurally globally identifiable.

\section{B.2 Identifiability of AMB-rotor model}

For grey-box identification in Step 4 of the procedure to work as intended, the parameter set $\left\{k_{S A}, k_{S B}, k_{i A}, k_{i B}\right\}$ has to be proven to be structurally identifiable for the model structure 
(2.12). The model in (2.12) includes both amplifier and AMB-rotor parts. Since the amplifier modes are identified separately and the parameter set in question does not have any connection to those modes, it is sufficient to prove identifiability for the AMB-rotor model (2.8).

Let the initial model be represented with state-space matrices which are parameterized with stiffness parameters only. Other parameters are assumed to be known and the real values are used here.

$$
\begin{gathered}
A(\theta)=\left[\begin{array}{cccc}
0 & 0 & 1 & 0 \\
0 & 0 & 0 & 1 \\
0.02 k_{S A} & -0.012 k_{S B} & 0 & 0 \\
-0.012 k_{S A} & 0.019 k_{S B} & 0 & 0
\end{array}\right] \\
B(\theta)=\left[\begin{array}{ccc}
0 & 0 \\
0 & 0 \\
0.02 k_{i A} & -0.012 k_{i B} \\
-0.012 k_{i A} & 0.019 k_{i B}
\end{array}\right] \\
C(\theta)=\left[\begin{array}{cccc}
1.16 & 0 & 0 & 0 \\
0 & 1.16 & 0 & 0
\end{array}\right]
\end{gathered}
$$

And the set of transformed model be represented by

$$
\begin{gathered}
A(\theta)=\left[\begin{array}{cccc}
0 & 0 & 1 & 0 \\
0 & 0 & 0 & 1 \\
0.02 \bar{k}_{s A} & -0.012 \bar{k}_{s B} & 0 & 0 \\
-0.012 \bar{k}_{S A} & 0.019 \bar{k}_{s B} & 0 & 0
\end{array}\right] \\
B(\theta)=\left[\begin{array}{ccc}
0 & 0 \\
0 & 0 \\
0.02 \bar{k}_{i A} & -0.012 \bar{k}_{i B} \\
-0.012 \bar{k}_{i A} & 0.019 \bar{k}_{i B}
\end{array}\right] \\
C(\theta)=\left[\begin{array}{cccc}
1.16 & 0 & 0 & 0 \\
0 & 1.16 & 0 & 0
\end{array}\right]
\end{gathered}
$$

Also, let the transformation matrix $T$ be

$$
T=\left[\begin{array}{llll}
t_{11} & t_{12} & t_{13} & t_{14} \\
t_{21} & t_{22} & t_{23} & t_{24} \\
t_{31} & t_{32} & t_{33} & t_{34} \\
t_{41} & t_{42} & t_{43} & t_{44}
\end{array}\right]
$$

From (B.4),

$$
\left[\begin{array}{cccc}
1.16 & 0 & 0 & 0 \\
0 & 1.16 & 0 & 0
\end{array}\right]=\left[\begin{array}{llll}
1.16 t_{11} & 1.16 t_{12} & 1.16 t_{13} & 1.16 t_{14} \\
1.16 t_{21} & 1.16 t_{22} & 1.16 t_{23} & 1.16 t_{24}
\end{array}\right]
$$




$$
\Leftrightarrow \quad \begin{aligned}
t_{11} & =t_{22}=1, \\
t_{12}=t_{13}=t_{14} & =t_{21}=t_{23}=t_{24}=0
\end{aligned}
$$

Updating this result into $T$ and from (B.2),

$$
\begin{aligned}
& {\left[\begin{array}{cccc}
t 31 & t 32 & t 33 & t 34 \\
t 41 & t 42 & t 43 & t 44 \\
0.02 \bar{k}_{S A} & -0.012 \bar{k}_{S B} & 0 & 0 \\
-0.012 \bar{k}_{S A} & 0.019 \bar{k}_{S B} & 0 & 0
\end{array}\right]} \\
& =\left[\begin{array}{cccc}
0 & 0 & 1 & 0 \\
0 & 0 & 0 & 1 \\
0.02 k_{S A} t_{33}-0.012 k_{S A} t_{34} & 0.02 k_{S B} t_{34}-0.012 k_{S B} t_{33} & t_{31} & t_{32} \\
0.02 k_{S A} t_{43}-0.012 k_{S A} t_{44} & 0.02 k_{S B} t_{44}-0.012 k_{S B} t_{43} & t_{41} & t_{42}
\end{array}\right] \\
& \Leftrightarrow \quad t_{33}=t_{44}=1 \text {, } \\
& t_{31}=t_{32}=t_{34}=t_{41}=t_{42}=t_{43}=0
\end{aligned}
$$

From (B.14) and (B.16),

$$
T=\left[\begin{array}{llll}
1 & 0 & 0 & 0 \\
0 & 1 & 0 & 0 \\
0 & 0 & 1 & 0 \\
0 & 0 & 0 & 1
\end{array}\right]
$$

Therefore, the parameter set is structurally identifiable for model (2.8). 


\section{Bibliography}

[1] H. Bleuler, E. H. Maslen, M. Cole, P. Keogh, R. Larsonneur, E. Maslen, R. Nordmann, Y. Okada, G. Schweitzer and A. Traxler, Magnetic Bearings: Theory, Design, and Application to Rotating Machinery, Springer, 2009.

[2] P. Förch, C. Gähler and R. Nordman, "AMB System for Rotordynamic Experiments: Calibration Results and Control," in Proceedings of the 5th International Symposium on Magnetic Bearings, Kanazawa, Japan, 1996.

[3] E. Maslen, "Magnetic Bearing Synthesis for Rotating Machinery," University of Virginia, USA, 1991.

[4] K. Yamashita, e. P. Allair and K. C., "Rotor Disturbance Attenuation Using An Hoo Controller for Active Magnetic Bearings," in Proceedings of the 5th International Symposium on Magnetic Bearings, Kanazawa, Japan, 1996.

[5] R. Fittro, C. Knospe and L. Stephens, "Experimental Results of $\mu$-Synthesis Applied to Point Compliance Minimization," in Proceedings of the 5th International Symposium on Magnetic Bearings, Kanazawa, Japan, 1996.

[6] F. Lösch, "Identification and Automated Controller Design for Active Magnetic Bearing Systems," ETH, Zurich, 2002.

[7] E. Lantto, "Robust Control of Magnetic Bearing in Subcritical Machines," Helsinki University of Technology, Helsinki, 1999.

[8] A. Smirnov, "AMB System for High-speed Motors uning Automatic Commissioning," Lappeenranta University of Technology, Lappeenranta, 2012.

[9] P. Beckerle, H. Schaede and S. Rinderknecht, "Model-Based Fault Detection on Active Magnetic Bearings by Means of Online Transfer-Factor Estimation," in Proc. of 13th International Symposium on Magnetic Bearings, Washington DC, 2012. 
[10] J. Sawicki, M. Friswell, Z. Kulesza, A. Wroblewski and J. Lekki, "Detecting cracked rotors using auxiliary harmonic excitation," Journal of Sound and Vibration, vol. 330, no. 7, p. $1365\{1381,2011$.

[11] B. Liu, J. Sjöberg and A. Laiho, "Optimization based AMB Controller Design and Verification for Flexible Rototrs," 2013.

[12] K. Hynynen, "Broadband Excitation in the System Identification of Active Magnetic Bearing Rotor Systems," Lappeenranta University of Technology, Lappeenranta, 2011.

[13] E. Wernholt, "Multivariable Frequency-Domain Identification of Industrial Robots," $\mathrm{PhD}$ thesis, Linköping University, Linköping, 2007.

[14] C. Gähler, M. Mohler and R. Herzog, "Multivariable Identification of Active Magnetic Bearing Systems," Springer, Netherlands, 1997.

[15] C. K. Sanathanan and J. Koerner, "Transfer Function Systhesis as a Ratio of Two Complex Polynomials," Automatic Control, IEEE Transactions on, 1963.

[16] R. S. Blom and M. J. V. d. Hof, "Multivariable Frequency Domain Identification using IV-based Linear Regression," in 49th IEEE Conference on Decision and Control, Atlanta, GA, USA, 2010.

[17] H.-J. Ahn, S.-W. Lee, S.-H. Lee and D.-C. Han, "Frequency Domain Control-relevant Identification of MIMO AMB rigid rotor," Automatica, 2003.

[18] H. M. N. K. Balini, I. Houtzager, J. Witte and C. W. Scherer, "Subspace and Frequency Domain Methods for Closed Loop Identification of AMB-Spindle," Stuttgart Research Centre for Simulation Technology, Stuttgart, 2011.

[19] R. Mohd-Mokhtar, L. Wang, L. Qin and T. Barry, "Continuous Time System Identification of Magnetic Bearing Systems Using Frequency Response Data," in 5th Asian Control Conference, 2004. 
[20] A. Chiuso, "On the relation between CCA and predictor-based subspace identi," IEEE Trans. on Automatic Control, pp. 1795-1812, 2007.

[21] L. Ljung, System Identification Theroy for the User, 2nd edition, New Jersey, USA: Prentice-Hall, 1999.

[22] Y. M. Cho, S. Srinavasan, J.-H. Oh and H. Soo, "Modelling and System Identification of Active Magnetic Bearing Systems," Mathematical and Computer Modelling of Dynamical Systems: Methods, Tools and Applications in Engineering and Related Sciences, 2007.

[23] R. Pintelon and J. Schoukens, System identification: a frequency domain approach, New York: IEEE Press, 2001.

[24] E. WaIter and L. Pronzato, Identification of Parametric Models, Springer, 1997.

[25] L. Ljung, " State of the art in linear system identification: Time and frequency domain methods," in American Control Conference, Boston, Massachusetts, 2004.

[26] T. McKelvey, "Identification of State-Space Models from Time and Frequency Data," Linköping University, Linköping, 1995.

[27] E. Wernholt and S. Moberg, "Experimental comparison of methods for multivariable frequency response function estimation," in 17th IFAC World Congress, Seoul, Korea, 2007.

[28] T. McKelvey, "Subspace methods for frequency domain data," in American Control Conference, Boston, MA, 2004.

[29] T. McKelvey, H. Akcay and L. Ljung, "Subspace-based multivariable system identification from frequency response data," IEEE Trans. on Automatic Control, vol. 41, no. 7, p. 960-979, 1996.

[30] U. Forssell, "Closed-loop Identification Methods, Theory, and Applications," Linkoping University, Linkoping, 1999. 
[31] L. Ljung, System Identification Toolbox User's Guide, MathWorks, 2014.

[32] B. Liu, ABB Corporate Research, Västerås, Sweden, Private communication, December 2014. 